\title{
Discovery of 21 New Changing-look AGNs in the Northern Sky
}

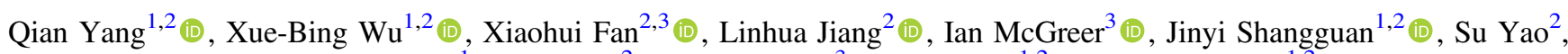 \\ Bingquan Wang ${ }^{1}$, Ravi Joshi ${ }^{2}$, Richard Green ${ }^{3}$, Feige Wang ${ }^{1,2}$ (D), Xiaotong Feng ${ }^{1,2}$, \\ Yuming $\mathrm{Fu}^{1,2}$, Jinyi Yang ${ }^{1,2}$ (D), and Yuanqi Liu ${ }^{1}$ \\ ${ }^{1}$ Department of Astronomy, School of Physics, Peking University, Beijing 100871, People's Republic of China; qianyang.astro@pku.edu.cn \\ ${ }^{2}$ Kavli Institute for Astronomy and Astrophysics, Peking University, Beijing 100871, People's Republic of China \\ ${ }^{3}$ Steward Observatory, University of Arizona, 933 North Cherry Avenue, Tucson, AZ 85721, USA \\ Received 2017 November 21; revised 2018 May 18; accepted 2018 June 2; published 2018 July 27
}

\begin{abstract}
The rare case of changing-look (CL) active galactic nuclei (AGNs), with the appearance or disappearance of broad Balmer emission lines within a few years, challenges our understanding of the AGN unified model. We present a sample of 21 new CL AGNs at $0.08<z<0.58$, which doubles the number of such objects known to date. These new CL AGNs were discovered in various ways, from (1) repeat spectra in the SDSS, (2) repeat spectra in the Large Sky Area Multi-Object Fiber Spectroscopic Telescope (LAMOST) and SDSS, and (3) photometric variability and new spectroscopic observations. We use the photometric data from surveys, including the SDSS imaging survey, the Pan-STARRS1, the DESI Legacy imaging survey, the Wide-field Infrared Survey Explorer (WISE), the Catalina Real-time Transient Survey, and the Palomar Transient Factory. The estimated upper limits of the transition timescale of the CL AGNs in this sample spans from 0.9 to 13 years in the rest-frame. The continuum flux in the optical and mid-infrared becomes brighter when the CL AGNs turn on, or vice versa. Variations of more than 0.2 mag in the $W 1$ band were detected in $15 \mathrm{CL}$ AGNs during the transition. The optical and mid-infrared variability is not consistent with the scenario of variable obscuration in $10 \mathrm{CL}$ AGNs at more than the $3 \sigma$ confidence level. We confirm a bluer-when-brighter trend in the optical. However, the mid-infrared WISE colors $W 1-W 2$ become redder when the objects become brighter in the $W 1$ band, possibly due to a stronger hot dust contribution in the $W 2$ band when the AGN activity becomes stronger. The physical mechanism of type transition is important for understanding the evolution of AGNs.
\end{abstract}

Key words: black hole physics - galaxies: active - galaxies: nuclei

\section{Introduction}

Active galactic nuclei (AGNs) are classified into Type 1 and Type 2 AGNs based on their emission-line features (e.g., Seyfert 1943; Khachikian \& Weedman 1971). Type 1 AGNs show broad (1000-20,000 $\mathrm{km} \mathrm{s}^{-1}$ ) and narrow (300-1000 $\mathrm{km} \mathrm{s}^{-1}$ ) emission lines, while only narrow emission lines are present in Type 2 AGNs (Netzer 2015, and references therein). This dichotomy is explained in the unified model (Antonucci 1993; Urry \& Padovani 1995) as a viewing angle effect due to the obscuration of the broad-line region (BLR). Intermediate AGN types exist. Type 1.8 (1.9) is classified by a broad $\mathrm{H} \alpha$ and a weak (absent) broad $\mathrm{H} \beta$ line (Osterbrock 1981). Type 1.5 is an intermediate type between Type 1 and Type 2 with an apparent narrow $\mathrm{H} \beta$ profile superimposed on broad $\mathrm{H} \beta$ components (Osterbrock \& Koski 1976; Osterbrock 1977). The intermediate type objects are explained as Type 2 objects observed in scattered light (Antonucci \& Miller 1985), or partial obscuration by optically thin dust (Stern \& Laor 2012). An alternative scenario is that the different classes are evolutionary (Penston \& Perez 1984; Korista \& Goad 2004; Wang \& Zhang 2007; Elitzur et al. 2014). Penston \& Perez (1984) proposed that the Type 2 class AGNs are possibly Type 1 AGNs in which the continuum source is temporarily off.

Some AGNs were observed to change between different spectral types; these are the so-called changing-look (CL) AGNs. The term "changing-look" was originally used in $\mathrm{X}$-rays, in which objects were found to change from Comptonthick to Compton-thin, or vice versa (e.g., Matt et al. 2003;
Bianchi et al. 2005; Piconcelli et al. 2007; Ballo et al. 2008; Risaliti et al. 2009; Marchese et al. 2012; Ricci et al. 2016). Lately, this term has been widened to describe objects with optical spectral type transitions (e.g., Denney et al. 2014; LaMassa et al. 2015; MacLeod et al. 2016; McElroy et al. 2016; Ruan et al. 2016; Runnoe et al. 2016; Gezari et al. 2017). The physical mechanisms of the changes are still under debate. The main plausible mechanisms are (1) variable obscuration due to the movement of obscuring material, in a scenario in which the dusty toroidal structure obscuring the BLR has a patchy distribution (e.g., Nenkova et al. 2008a, 2008b; Elitzur 2012) or an accelerating outflow (e.g., Shapovalova et al. 2010); (2) variable accretion rate, in an evolutionary paradigm in which an AGN follows an evolutionary sequence from Type 1 to intermediate type and later to Type 2, or vice versa (e.g., Penston \& Perez 1984; Elitzur et al. 2014); or (3) a tidal disruption event (TDE) of a star disrupted by the supermassive black hole (SMBH), which may also result in a change of classification (Eracleous et al. 1995; Merloni et al. 2015; Blanchard et al. 2017). The nature of the type transition is important for understanding the evolution of AGNs.

Spectral type changes, with the appearance or disappearance of broad Balmer emission lines, have been detected in only a small number of AGNs. Long-timescale observations of a handful of AGNs show that some AGNs have changed from Type 1 to Type 2, and back and forth. Mrk 1018 had changed from a Type 1.9 to a Type 1 Seyfert, and changed back to Type 1.9 after 30 years (Cohen et al. 1986; McElroy et al. 2016). Observations over more than 40 years reveal that Mrk 590 
changed from Type 1.5 to Type 1 and changed back to Type 1.9-2 (Denney et al. 2014). NGC 2617, which was a Seyfert 1.8 galaxy (Moran et al. 1996), changed to a Seyfert 1 (Shappee et al. 2014). These cases are better explained as changes in luminosity rather than obscuration (Denney et al. 2014; Shappee et al. 2014; McElroy et al. 2016). The broad emission lines of NGC 4151, which was originally Type 1.5 (Osterbrock 1977), had once disappeared (Antonucci \& Cohen 1983; Lyutyj et al. 1984; Penston \& Perez 1984) and then returned (e.g., Shapovalova et al. 2010). The variation in NGC 4151 is explained as probably being caused by an accelerating outflow originating very close to the black hole $(\mathrm{BH}$; Shapovalova et al. 2010). Such back-and-forth type changes encourage exploration of what mechanisms generate these changes.

In recent years, discoveries of CL quasars have shown that such transitional phenomena happen at higher redshifts and can occur in more luminous and massive systems. Until now, there were fewer than 20 known CL quasars. LaMassa et al. (2015) reported the first CL quasar, J0159+0033, which changed from Type 1 to Type 1.9. They demonstrated that variable absorption does not explain the observed timescales and large-scale obscuration material is needed for this scenario. Merloni et al. (2015) argued that J0159+0033 could be a luminous flare produced by a TDE. Runnoe et al. (2016) reported a CL quasar, $\mathrm{J} 1011+5442$, "turning-off" within a rest-frame time of approximately 500 days. They argued that the transition timescale is inconsistent with an abrupt change in the reddening toward the central engine, and the decaying light curve with a prolonged bright state preceding the decay is not consistent with a decaying TDE. If the type changes were caused by the obscuration of the quasar, high linear polarization would be expected (Hutsemékers et al. 2017; Marin 2017). Hutsemékers et al. (2017) measured the polarization of $\mathrm{J} 1011+5442$, and found null polarization, suggesting that the type transition was not due to variable obscuration. A rapid "turn-on" of a quasar, $\mathrm{J} 1554+3629$, was detected by iPTF on a timescale of less than one year (Gezari et al. 2017). They implied that a factor of 10 brightening in UV and X-ray continuum flux is more likely caused by an intrinsic change in the accretion rate. Some systematic archival searches for objects in the Sloan Digital Sky Survey (SDSS) with repeat spectroscopy found more CL quasars (MacLeod et al. 2016; Ruan et al. 2016). They implied that changes in the accretion rate can better explain the transition timescale and emission-line properties than variable dust obscuration. Sheng et al. (2017) argued that the large variability amplitude of CL AGNs in the mid-infrared supports the scenario in which changes in the accretion rate occurs for reasons other than varying obscuration. In a recent TDE (PS16dtm), broad Balmer emission lines appeared to be accompanied by a significant increase in the continuum flux, as well as strong Fe II and He II emissions (Blanchard et al. 2017).

The frequency and timescale of such transients in the universe are interesting issues. Martini \& Schneider (2003) suggested that the number of turn-off quasars in a large number of quasars can be used to measure or set a lower limit on the episodic lifetime of quasars. In the search by MacLeod et al. (2016), out of more than 1000 quasars with $g$-band variability larger than one mag, only 10 objects showed variable broad emission lines (MacLeod et al. 2016). The majority of highly variable quasars did not exhibit emerging or disappearing broad emission lines. Rumbaugh et al. (2018) identified $\sim 1000$ extreme variability quasars (EVQs) with a maximum $g$-band magnitude change of more than one mag with the SDSS and 3 Year Dark Energy Survey (DES, Flaugher 2005) imaging. They claimed that these EVQs are good candidates for CL AGNs.

CL AGNs provide perfect cases to study the connection between AGNs and their host galaxies. There is a tight correlation between $\mathrm{BH}$ mass, $M_{\mathrm{BH}}$, and the velocity dispersion, $\sigma_{*}$, of the bulge component in nearby galaxies (Kormendy \& Ho 2013). Gezari et al. (2017) reported that the $M_{\mathrm{BH}}\left(2_{-1.5}^{+4} \times 10^{8} M_{\odot}\right)$ of $\mathrm{J} 1554+3629(z=0.237)$, estimated from the quasar spectrum after "turning on" with emerging broad Balmer emission lines, is in good agreement with its $M_{\mathrm{BH}}\left(1_{-0.7}^{+2} \times 10^{8} M_{\odot}\right)$ inferred from $\sigma_{*}$. CL AGNs provide exceptional opportunities to provide the central BHs and their host galaxies at higher redshift. On the other hand, the "turning-off" CL quasars provide perfect opportunities to study the host galaxies of quasars in detail, avoiding contamination from the luminous central engines.

The CL AGNs, with the appearance or disappearance of broad Balmer lines on a timescale of years, challenge our understanding of the AGN unification model. Motivated by an interest in determining the physical mechanism behind type transitions, and questions regarding the frequency and timescale of the type transition, we conduct a survey for CL AGNs. The investigations consist of (1) repeat spectroscopy in the SDSS spectral archive, (2) repeat spectroscopy in the SDSS and the Large Sky Area Multi-Object Fiber Spectroscopic Telescope (LAMOST) spectral archive, and (3) searching for CL candidates based on photometric variability.

This paper is organized as follows. Section 2 describes the spectroscopic and imaging data. Section 3 outlines the selection methods and spectroscopic observations. In Section 4, we present new CL AGNs and their variability in the optical and mid-infrared. In Section 5, we discuss the CL AGN color variability and the timescale of the type transition. We summarize the paper in Section 6. In this work we adopt a standard $\Lambda$ CDM cosmology with $\Omega_{\Lambda}=0.7, \Omega_{m}=0.3$, and $H_{0}=70 \mathrm{~km} \mathrm{~s}^{-1} \mathrm{Mpc}^{-1}$. Throughout this paper, all magnitudes are in $\mathrm{AB}$ magnitudes.

\section{Data \\ 2.1. Spectroscopic Data \\ 2.1.1. SDSS Spectroscopy}

There are 4,851,200 spectra in the SDSS Fourteenth Data Release (DR14; Abolfathi et al. 2017) taken by the Sloan Foundation $2.5 \mathrm{~m}$ telescope (Gunn et al. 2006) at Apache Point Observatory. The spectra are from the SDSS-I/II, with a wavelength coverage from 3800 to $9100 \AA$, and the Baryon Oscillation Spectroscopic Survey (BOSS; Dawson et al. 2013) spectrograph of the SDSS-III (Eisenstein et al. 2011), with a wavelength coverage from 3600 to $10400 \AA$ (Smee et al. 2013). The spectral resolution is 1500 at $3800 \AA$ and 2500 at $9000 \AA$. The SDSS spectroscopic pipelines classify the objects as galaxies ("GALAXY"), stars ("STAR"), or quasars ("QSO"), through a comparison of individual spectra with galaxy, QSO, and stellar templates (Bolton et al. 2012; Hutchinson et al. 2016). The spectral quality is described by a confidence flag titled "zWarning," which is 0 or 16 for good data without identified problems (e.g., Stoughton et al. 2002). We carry out searches of CL AGNs from SDSS galaxies or quasars. 


\subsubsection{LAMOST Spectroscopy}

LAMOST is a $4 \mathrm{~m}$ reflecting Schmidt telescope equipped with 4000 fibers with a $5^{\circ}$ field of view (Cui et al. 2012; Zhao et al. 2012). The wavelength coverage of LAMOST ranges from $3700 \AA$ to $9000 \AA$, with two arms (Du et al. 2016), a blue

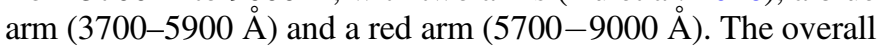
spectral resolution of LAMOST is approximately 1800 . The data are reduced with LAMOST pipelines (Luo et al. 2012). In this paper, we utilize the LAMOST spectra from data releases one to five (Luo et al. 2015; He et al. 2016).

\subsection{Imaging Data}

CL AGNs showed continuum flux changes in the optical and mid-infrared. Apart from objects with repeat spectroscopy, we carry out additional searches of CL AGNs based on imaging data. We briefly introduce the imaging data as follows.

The SDSS imaging survey scanned the sky in five filters, ugriz (Fukugita et al. 1996), covering 11,663 $\mathrm{deg}^{2}$ in SDSS-I/ II from 2000 to 2007 (Abazajian et al. 2009) and an additional 3,000 $\mathrm{deg}^{2}$ in SDSS-III in 2008.

The Pan-STARRS1 (PS1; Chambers et al. 2016) survey used a 1.8-meter telescope with a 1.4 Gigapixel camera to image the sky in five broadband filters (grizy). The observations cover three-quarters of the sky several times per filter. We use the PS1 magnitudes in the stack catalog, from co-added images made from the multiple exposures. Using a quasar composite spectrum from Vanden Berk et al. (2001) convolved with the PS1 and SDSS $g$-band filter curves (Equation (2) in Wu et al. 2004), the magnitude difference, $\delta\left(g_{\mathrm{PS} 1}-g_{\text {SDSS }}\right)$, is between $-0.065 \mathrm{mag}$ and $0.008 \mathrm{mag}$ at redshift $z<2$.

The DESI Legacy imaging survey (DELS; Dey et al. 2018) commenced imaging surveys, including the DECam Legacy Survey (DECaLS) $g-, r$-, and $z$-bands, the $g$ - and $r$-bands of the Beijing-Arizona Sky Survey (BASS; Zou et al. 2017), and the Mayall $z$-band Legacy Survey (MzLS). The imaging survey commenced from 2014 and will conclude in 2018, covering $14000 \mathrm{deg}^{2}$ sky. The magnitude difference, $\delta\left(g_{\text {DELS }}-g_{\text {SDSS }}\right)$, is between $-0.053 \mathrm{mag}$ and $0.005 \mathrm{mag}$ at $z<2$, using the composite spectrum convolved with the DELS and SDSS $g$ band filter curves.

The Wide-field Infrared Survey Explorer (WISE; Wright et al. 2010) mapped the all-sky from 2010 January to July in four bands centered at wavelengths of 3.4, 4.6, 12, and $22 \mu \mathrm{m}$ $(W 1, W 2, W 3$, and $W 4)$. The secondary cryogen survey and Near-Earth Object Wide-field Infrared Survey Explorer (NEOWISE; Mainzer et al. 2011) Post-Cryogenic Mission mapped the sky from 2010 August to 2011 February. The NEOWISE Reactivation Mission (NEOWISE-R; Mainzer et al. 2014) surveys the sky in the $W 1$ and $W 2$ bands from 2013. We use WISE multi-epoch photometry from individual single-exposure images in the $W 1$ and $W 2$ bands.

The Catalina Real-time Transient Survey (CRTS; Drake et al. 2009) uses data from the Catalina Sky Survey, which repeatedly covers $26,000 \mathrm{deg}^{2}$ on the sky. The CRTS photometric data are unfiltered and calibrated to $V$-band magnitude. We apply a constant offset to the CRTS magnitudes to match the simultaneous $g$-band PS1 magnitude.

The Palomar Transient Factory (PTF; Law et al. 2009) is a wide-field survey covering approximately $30,000 \mathrm{deg}^{2}$ in the $g$ - and $r$ - bands from 2009 to 2012.

\section{Target Selection and Observation}

\subsection{SDSS Repeat Spectroscopy}

We carried out a systematic investigation in the SDSS DR14 spectral archive, in which $87 \%$ of spectra are good quality (zWarning $=0$ or 16). Cross-matching the spectra with a radius of $2^{\prime \prime}$ results in 350,609 objects repeatedly observed $(\geqslant 2$ epochs $)$. There were 175,575 repeatedly observed objects classified as "GALAXY" by the SDSS pipeline in at least one epoch spectrum. Among them, 2,023 objects were classified as "GALAXY" in one epoch of the spectrum and "QSO" in another epoch of the spectrum. We visually checked all the spectra of these objects to search for CL AGNs.

Because the definitions of AGN spectral types depend on the strength of broad emission lines, we qualitatively define a detection of a broad emission line if the signal-to-noise ratio $(\mathrm{S} / \mathrm{N})$ of the emission line is higher than 5 or as a non-detection if the $\mathrm{S} / \mathrm{N}$ is lower than 1 . Specifically, a weak detection is when the $\mathrm{S} / \mathrm{N}$ is between 1 and 3 , and an intermediate detection is when the $\mathrm{S} / \mathrm{N}$ is between 3 and 5 . Visual checks cannot accurately discern the quantitative changes of emission lines, but they can qualitatively distinguish the cases with dramatic changes. More specifically, with a visual inspection it is easier to distinguish $>5 \sigma$ and $<1 \sigma$ detections than to distinguish $3 \sigma-5 \sigma$ and $1 \sigma-3 \sigma$ detections. Therefore, the visual inspection process is inclined to select AGNs that changed between Type 1 and Type 2 (or Type 1.9), with an appearing or disappearing broad $\mathrm{H} \alpha$ (or $\mathrm{H} \beta$ ) emission line. Nevertheless, a visual check selects AGNs that changed between Type 1 and Type 1.8 with a lower completeness. Objects with distinct companions (within $2^{\prime \prime}$ ) in the SDSS image were also excluded in the visual check process. From the visual inspection, we selected 9 CL AGNs. Four of the selected CL AGNs were reported previously, including the CL quasar J0159+0033 in LaMassa et al. (2015), J0126-0839 and J2336+0017 in Ruan et al. (2016), and J1011+5442 in Runnoe et al. (2016). We recovered all the changing-look quasars in these three works. The new CL AGNs found in the SDSS archive are J1104 +6343 , J1118+3203, J1150+3632, J1358+4934, and $\mathrm{J} 1533$ +0110 . We describe the objects rejected by visual inspection in the Appendix. In Section 4.1, we describe the fitting of the $\mathrm{H} \alpha$ and $\mathrm{H} \beta$ lines of these objects to quantitatively understand the changes of CL AGNs selected from visual inspection. The ratio of CL AGNs with an appearance or disappearance of broad Balmer emission lines is roughly $0.006 \%$, for objects that were identified as galaxies and repeatedly observed. This ratio may be affected by some issues, for example, the time intervals of the repeat spectra, spectroscopic survey selection bias, spectral quality, and possible selection bias of visual inspection. The selection steps are summarized in Table 1.

\subsection{LAMOST and SDSS Repeat Spectroscopy}

We cross-matched the LAMOST spectral archive with the SDSS spectra archive. The selection steps are summarized in Table 2. There were 155,220 objects that were classified as "GALAXY" (75\%) or "QSO" (25\%) with good spectral quality in the SDSS, or were observed in LAMOST. Comparing the SDSS spectra with the LAMOST spectra, we fit the Balmer emission lines in spectra from the SDSS and the LAMOST with Gaussian profiles. We selected the objects with changes in the emission-line flux density larger than $2 \times 10^{-18} \mathrm{erg} \mathrm{s}^{-1} \mathrm{~cm}^{-2} \AA^{-1}$, which is a conservative criterion 
Table 1

CL AGNs Selection from SDSS Repeat Spectra

\begin{tabular}{|c|c|c|}
\hline Note & Selection & Number \\
\hline Spectra in SDSS DR14 & All & $4,851,200$ spectra \\
\hline Spectra with good quality & $z$ Warning $=0$ or 16 & $4,196,290$ spectra \\
\hline Objects with repeat spectra & $2^{\prime \prime}$ coordinates cross-match & 350,609 objects \\
\hline Galaxies with repeat spectra & classified as "GALAXY" at one epoch & 175,575 objects \\
\hline Classification changed between QSO and Galaxy & classified as “QSO” at another epoch & 2,023 objects \\
\hline Visual check & appearing or disappearing broad $\mathrm{H} \beta$ & 9 (4 known) CL AGNs \\
\hline
\end{tabular}

Table 2

CL AGNs Selection from SDSS and LAMOST Repeat Spectra

\begin{tabular}{|c|c|c|}
\hline Note & Selection & Number \\
\hline Spectra with good quality & $z$ Warning $=0$ or 16 & $4,196,290$ spectra \\
\hline SDSS QSO/Galaxy & classified as "GALAXY" or "QSO" & $3,223,478$ spectra \\
\hline Repeatedly observed by LAMOST & $2^{\prime \prime}$ cross-match with LAMOST & 155,220 objects \\
\hline Possible variable Balmer lines & program to automatically check emission-line variation & 8,181 objects \\
\hline Visual check & appearing or disappearing broad $\mathrm{H} \beta$ & $10 \mathrm{CL}$ AGNs \\
\hline
\end{tabular}

avoiding missing CL AGNs at this step. There were 8,181 objects with potential emission-line variation. We visually inspected all these objects, and identified $10 \mathrm{CL}$ AGNs, including 8 turn-on CL AGNs (J0831+3646, J0909+4747, $\mathrm{J} 0937+2602$, J1115+0544, J1132+0357, J1447+2833, J1545 +2511 , and J1552+2737) and 2 turn-off AGNs (J0849+2747 and $\mathrm{J} 1152+3209)$. We obtained flux calibrations to match the narrow emission-line flux of the SDSS spectra, assuming a constant narrow emission-line flux within a few tens of years. We describe the objects visually rejected in the Appendix. In this survey, about $0.007 \%$ of the galaxies from the LAMOST and SDSS cross-matched sample were proven to be CL AGNs with the appearance of broad Balmer emission lines.

\subsection{Photometric Variability}

Most of the reported CL AGNs were discovered by repeat spectroscopy. Meanwhile, the optical and mid-infrared flux of CL AGNs varies following the type transition. We conducted additional CL AGN searches based on photometric variability. We searched CL AGN candidates from objects that were spectroscopically identified as "GALAXY" but brightened in later photometric data, and from objects that were spectroscopically identified as "QSO" but significantly dimmed.

\subsubsection{Turn-on CL AGNs Selected from Imaging Data}

We carried out a search for turn-on CL AGNs from SDSS galaxies, which became brighter later. The selection procedures are shown in Table 3. Motivated by the rapid transition that happened in $\mathrm{J} 1011+5442$ and $\mathrm{J} 1554+3629$, we first considered recent imaging data. The WISE multi-epoch data are available for recent years, from 2010 to 2017 . We started from galaxies detected by WISE multi-epoch data, and there were 28,395 galaxies that became brighter $(\Delta W 1<-0.2 \mathrm{mag})$ and redder $(\Delta(W 1-W 2)>0.1 \mathrm{mag})$ in the mid-infrared. A more detailed discussion about the color criteria in the mid-infrared will be provided in Section 5.1. Among them, 2147 galaxies also became brighter in the optical $g$-band and had $g<19$ mag. We checked the CRTS and PTF light curves of these galaxies at $z>0.1$, and picked out 59 turn-on CL AGN candidates with a clear trend of increasing flux in the optical and mid-infrared. This selection method recovered J0831 +3646 and J1554+3629. Other turn-on CL AGNs from repeat spectroscopy were missed in this process due to smaller redshift or smaller variability. More systematic searches can be extended to lower redshifts and fainter objects with less variability.

Optical long-slit spectroscopic observations for some CL AGN candidates were carried out using the Xinglong $2.16 \mathrm{~m}$ telescope (XLT) in China and Palomar P200/DBSP spectrograph. The Xinglong $2.16 \mathrm{~m}$ telescope is located at the NAOC observatory. It is equipped with the Beijing Faint Object Spectrograph and Camera (BFOSC). We observed 17 CL AGN candidates in 2017 April, including a CL AGN J1554+3629 discovered by Gezari et al. (2017), using the BFOSC and Grism 4 (G4) with a dispersion of $198 \AA / \mathrm{mm}$ and a wavelength coverage from 3850 to $8300 \AA$. The grism yields a resolution of $R \sim 265$ or 340 at $5000 \AA$ using a 2 !" 3 or 1 !" 8 slit (Fan et al. 2016). The spectra were obtained using a 1"! 8 slit when seeing $<2^{\prime \prime}$ or a $2^{\prime \prime} 3$ slit when $2^{\prime \prime}<$ seeing $<3^{\prime \prime}$. J1259 +5515 was observed by DBSP after it was observed by XLT. The DBSP spectrum was obtained using P200/DBSP with grating $\mathrm{G} 600$ at the blue side $(R \sim 1200$ at $5000 \AA)$ and $\mathrm{G} 316$ at the red side $(R \sim 642$ at $5000 \AA)$ with a 1 ." 5 slit under seeing $\sim 1$ !' 5 . The spectra were reduced using standard IRAF ${ }^{4}$ routines (Tody 1986, 1993). The flux of J1259+5515 obtained with DBSP and XLT in two days are in good agreement. Five new CL AGNs (J1003+3525, J1110-0003, J1259+5515, J1319 +6753 , and $\mathrm{J} 1550+4139)$, with emerging broad $\mathrm{H} \beta$, were confirmed by the XLT and DBSP spectra. The fiber diameters of SDSS, BOSS, and LAMOST are $3^{\prime \prime}, 2^{\prime \prime}$, and 3."3, respectively. The fiber diameters are large enough to include the light from the nuclear. The slit widths of long-slit spectra are slightly smaller than the fiber diameters, leading to less host galaxy light contributions to the spectra. The photometric variability of these CL AGNs rules out the possibility that the different spectra are merely caused by differences between fiber and slit spectra.

\footnotetext{
4 IRAF is distributed by the National Optical Astronomy Observatory, which is operated by the Association of Universities for Research in Astronomy (AURA) under cooperative agreement with the National Science Foundation.
} 
Table 3

Turn-on CL AGN Candidate Selection Based on Imaging Data

\begin{tabular}{|c|c|c|}
\hline Note & Selection & Number \\
\hline Spectra in SDSS DR14 & all & $4,851,200$ spectra \\
\hline WISE single-epoch detected & $2^{\prime \prime}$ cross-match with the WISE single-epoch data & $4,196,290$ spectra \\
\hline Galaxies with good spectra & class $="$ GALAXY" and (zWarning $=0$ or 16$)$ & $2,494,319$ spectra \\
\hline WISE brighten and redder & $\Delta W 1<-0.2$ and $\Delta(W 1-W 2)>0.1$ & 28,395 objects \\
\hline Optical brighten & $\Delta g<0$ and $g<19$ & 2147 objects \\
\hline Redshift & $z>0.1$ & 660 objects \\
\hline Visual check light curves & obvious brighten trend (CRTS/PTF) & 59 objects \\
\hline Observed & $\cdots$ & 17 objects \\
\hline Confirmed & $\cdots$ & 6 (1 known) CL AGNs \\
\hline
\end{tabular}

Table 4

Turn-off CL AGN Candidate Selection Based on Imaging Data

\begin{tabular}{lcc}
\hline \hline Note & Selection & Number \\
\hline SDSS QSO in DR7 & All & 346,464 \\
and DR12 & & objects \\
WISE single-epoch & $2^{\prime \prime}$ cross-match with the WISE single- & 326,124 \\
$\quad$ detected & epoch photometry & objects \\
WISE dim and bluer & $\Delta W 1>0.2$ and $\Delta$ & 6,847 objects \\
& $(W 1-W 2)<-0.1$ & 232 objects \\
Optical dim & $\Delta g>1$ & 1 objects \\
Observed & $\ldots$ & 1 CL AGN c \\
Confirmed & $\ldots$ &
\end{tabular}

\subsection{Turn-off CL AGNs Selected from Imaging Data}

We conducted a survey of turn-off CL AGNs from SDSS quasar catalogs (shown in Table 4). There are 346,464 quasars (Yang et al. 2017) in the SDSS Data Release 7 Quasar catalog (Schneider et al. 2010) and the Data Release 12 Quasar catalog (Pâris et al. 2017). Cross-matching with a radius of 2" ,94\% of quasars were detected in the WISE multi-epoch data. There were 6,847 quasars that dimmed $(\Delta W 1>0.2 \mathrm{mag})$ and became bluer $(\Delta(W 1-W 2)<-0.1 \mathrm{mag})$ in the mid-infrared.

On a timescale of months to years, the continuum variability of quasars is typically $0.2 \mathrm{mag}$ in the optical (e.g., Vanden Berk et al. 2004; Wilhite et al. 2005; MacLeod et al. 2012). Among the quasars with mid-infrared variability, 232 quasars also dimmed significantly in the optical (more than 1 mag in the $g$ band). We recovered two turn-off CL AGNs, including J0849 +2747 and J1011+5442. In 2017 April, we observed one candidate (with the XLT using a 2!"3 slit), J1104+0118, with $g$-band and W1-band magnitudes dimmed by more than 1.8 mag and 0.6 mag, respectively. The continuum flux of J1104 +0118 obviously became much redder than that in the SDSS spectrum, and the broad Mg II emission line was not detected in the new spectrum. Higher-quality spectra are needed for a more detailed analysis A more complete survey of CL AGNs will require more spectroscopic follow-up.

\section{Results}

\subsection{New CL AGNs}

In total, there are 21 new CL AGNs found in our surveys (summarized in Table 5). Among the new CL AGNs, 5 were found by repeat spectra from the SDSS, 10 were discovered based on repeat spectra from SDSS and LAMOST, and 6 were selected from photometric variability in optical and midinfrared imaging data, and confirmed by new spectroscopy.
The five known CL AGNs that we recovered are also listed in Table 5. Figure 1 shows an example of the light curves (left panels), and spectra (right panels) of a new CL AGN, J0831 +3646 . The light curves and spectra of the other 20 new CL AGNs are listed in the Appendix. In Figure 1, the spectra separating for 13 years show that there was no $\mathrm{H} \beta$ emission in 2002 in the SDSS (black), while the broad $\mathrm{H} \beta$, as well as broad $\mathrm{H} \alpha$, emission lines emerged in the spectra taken in 2015 in LAMOST (blue). The residual spectrum (gray spectrum at the bottom of the right panel), between the bright epoch and faint epoch spectra, distinctly shows the emerging of broad $\mathrm{H} \beta$ and $\mathrm{H} \alpha$. The left panel in Figure 1 shows the photometric data from several surveys, including SDSS, PS1, DELS, WISE, CRTS, and PTF. The imaging data taken in separated epochs help identify the variability of objects.

We fit the spectra of all the CL AGNs with a quasar spectral fitting procedure (QSfit, Calderone et al. 2017), considering the contribution from the AGN continuum, Balmer continuum, host galaxy, blended iron lines, broad emission lines, and narrow emission lines. The spectroscopic measurements are summarized in Table 6 . The later epoch spectrum is calibrated to the earlier SDSS spectrum assuming a constant narrow emission line during a few years. Only in 4 out of these 21 objects is the $\mathrm{S} / \mathrm{N}$ of the faint-state spectrum lower than that of the bright state spectrum. The weaker Balmer emission-line intensity at the faint state is not a result of a worse observational condition.

According to the definition of AGN spectral types, we mark the spectral type as Type 1 when there are $(>5 \sigma)$ broad $\mathrm{H} \beta$ components detected and Type 2 when the $\mathrm{S} / \mathrm{N}$ of the broad $\mathrm{H} \alpha$ is lower than 5. Specifically, when $(>5 \sigma) \mathrm{H} \alpha$ is detected, we mark the spectal type as Type $1.9(1.8$ or 1.5$)$ when there is no, $<1 \sigma$, (weak, $1 \sigma-3 \sigma$, or intermediate, $3 \sigma-5 \sigma$ ) broad $\mathrm{H} \beta$ detected. As the Balmer emission lines disappeared in some spectra, we freed the parameter limit of the amplitude of the line fitting, which was assumed to be positive. The fitting results are shown in Table 6 . The negative luminosity means that there is absorption instead of emission lines. As shown in Table 6, six CL AGNs transitioned between Type 1 and Type 2 classes, and $10 \mathrm{CL}$ AGNs transitioned between Type 1 and Type 1.9 classes. In addition, the fitting procedure recognizes $>5 \sigma$ broad $\mathrm{H} \beta$ emission lines in both bright and faint-state spectra of $\mathrm{J} 1118+3203$ and $\mathrm{J} 1152+3209$, and $<1 \sigma$ broad $\mathrm{H} \beta$ lines in both bright and faint-state spectra of $\mathrm{J} 1552+2737$. Therefore, these three AGNs fail to fit the quantitative definition above. While the dramatic $\mathrm{H} \beta$ luminosity changes show that $\mathrm{H} \beta$ faded in $\mathrm{J} 1118+3203$ and $\mathrm{J} 1152+3209$ (marked as "off"" in Table 6) and enhanced in J1152+3209 (marked as "on"" in Table 6). Among them, 15 AGNs turned on, with 

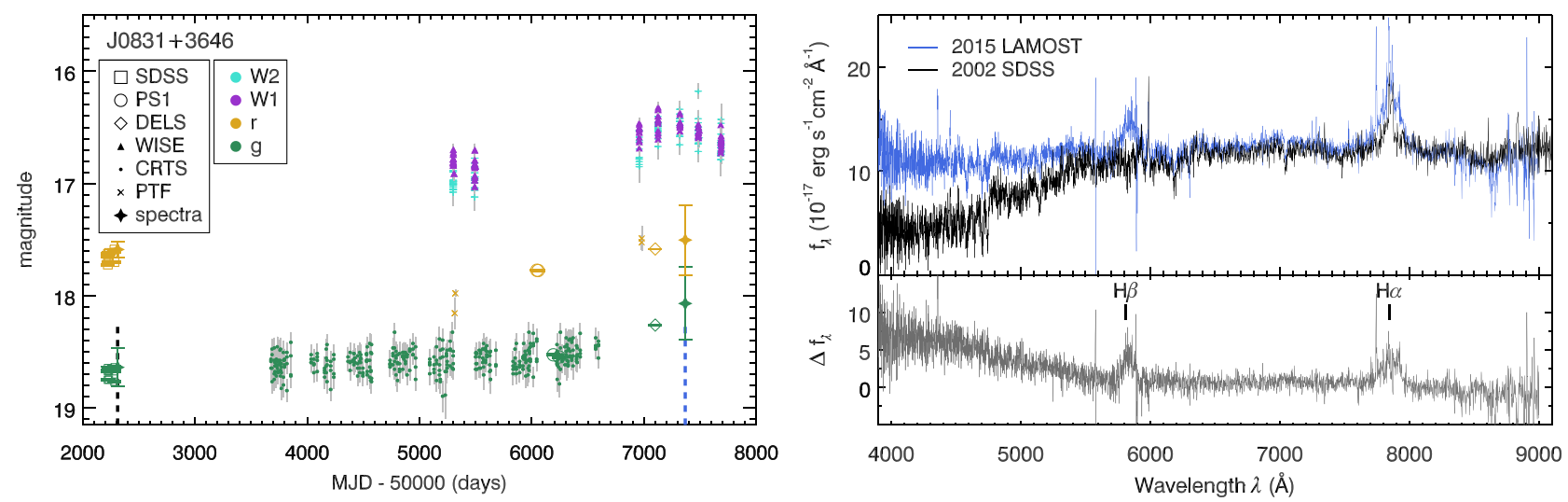

Figure 1. Example of the light curves (left panel) and spectra (right panel) of a new CL AGN, J0831+3646. In the left panel, all photometric data are expressed in magnitude, and the $x$-axis is MJD-50000. Photometric data in distinct bands are shown in different colors, including the $g$ (green), $r$ (yellow), $W 1$ (magenta), and $W 2$ (cyan) band photometry. The different shapes represent data from different surveys, including SDSS (open square), PS1 (open circle), DELS (open diamond), WISE W1 (solid triangle), WISE W2 (cross), CRTS (solid dots), and PTF (x shape). The vertical dashed lines show the epochs of the spectra. The right panel shows the spectral flux density, $f_{\lambda}$, of CL AGNs in units of $10^{-17} \mathrm{erg} \mathrm{s}^{-1} \mathrm{~cm}^{-2} \AA^{-1}$. The early-epoch SDSS spectrum is plotted in black, and the new epoch spectrum is colored blue if the AGN turned on, or red if the AGN turned off. The lower panel shows the spectra difference, $\Delta f_{\lambda}$, between the bright epoch and faint epoch spectra. The vertical bars mark the locations of appeared or disappeared broad emission lines. The light curves and spectra of the other 20 CL AGNs are in the Appendix.

Table 5

CL AGNs

\begin{tabular}{|c|c|c|c|c|c|c|c|}
\hline Name & R.A. & Decl. & Redshift & Transition & Epoch & Instrument2 & References \\
\hline J0831+3646 & $08: 31: 32.25$ & $+36: 46: 17.2$ & 0.19501 & Turn-on & {$[52312,57367]$} & LAMOST & This work \\
\hline $\mathrm{J} 0849+2747$ & 08:49:57.78 & $+27: 47: 28.9$ & 0.29854 & Turn-off & {$[53350,56628]$} & LAMOST & This work \\
\hline J0909+4747 & 09:09:32.02 & $+47: 47: 30.6$ & 0.11694 & Turn-on & {$[52620,57745]$} & LAMOST & This work \\
\hline $\mathrm{J} 0937+2602$ & 09:37:30.32 & $+26: 02: 32.1$ & 0.16219 & Turn-on & {$[54524,57369]$} & LAMOST & This work \\
\hline $\mathrm{J} 1003+3525$ & $10: 03: 23.47$ & $+35: 25: 03.8$ & 0.11886 & Turn-on & {$[53389,57867]$} & XLT & This work \\
\hline $\mathrm{J} 1104+0118$ & 11:04:55.17 & $+01: 18: 56.6$ & 0.57514 & Turn-off & {$[52374,57867]$} & XLT & This work \\
\hline $\mathrm{J} 1104+6343$ & $11: 04: 23.21$ & $+63: 43: 05.3$ & 0.16427 & Turn-off & {$[52370,54498]$} & SDSS & This work \\
\hline $\mathrm{J} 1110-0003$ & $11: 10: 25.44$ & $-00: 03: 34.0$ & 0.21922 & Turn-on & {$[51984,57864]$} & XLT & This work \\
\hline $\mathrm{J} 1115+0544$ & $11: 15: 36.57$ & $+05: 44: 49.7$ & 0.08995 & Turn-on & {$[52326,57393]$} & LAMOST & This work \\
\hline $\mathrm{J} 1118+3203$ & $11: 18: 29.64$ & $+32: 03: 59.9$ & 0.3651 & Turn-off & {$[53431,56367]$} & BOSS & This work \\
\hline $\mathrm{J} 1132+0357$ & $11: 32: 29.14$ & $+03: 57: 29.0$ & 0.09089 & Turn-on & {$[52642,57392]$} & LAMOST & This work \\
\hline $\mathrm{J} 1150+3632$ & $11: 50: 39.32$ & $+36: 32: 58.4$ & 0.34004 & Turn-off & {$[53436,57422]$} & BOSS & This work \\
\hline $\mathrm{J} 1152+3209$ & $11: 52: 27.48$ & $+32: 09: 59.4$ & 0.37432 & Turn-off & {$[53446,57844]$} & LAMOST & This work \\
\hline $\mathrm{J} 1259+5515$ & $12: 59: 16.74$ & $+55: 15: 07.2$ & 0.19865 & Turn-on & {$[52707,57863]$} & XLT/DBSP & This work \\
\hline $\mathrm{J} 1319+6753$ & $13: 19: 30.75$ & $+67: 53: 55.4$ & 0.16643 & Turn-on & {$[51988,57867]$} & XLT & This work \\
\hline $\mathrm{J} 1358+4934$ & 13:58:55.82 & $+49: 34: 14.1$ & 0.11592 & Turn-on & {$[53438,54553]$} & SDSS & This work \\
\hline $\mathrm{J} 1447+2833$ & $14: 47: 54.23$ & $+28: 33: 24.1$ & 0.16344 & Turn-on & {$[53764,57071]$} & LAMOST & This work \\
\hline $\mathrm{J} 1533+0110$ & 15:33:55.99 & $+01: 10: 29.7$ & 0.14268 & Turn-on & {$[51989,54561]$} & SDSS & This work \\
\hline $\mathrm{J} 1545+2511$ & $15: 45: 29.64$ & $+25: 11: 27.9$ & 0.11696 & Turn-on & {$[53846,57891]$} & LAMOST & This work \\
\hline $\mathrm{J} 1550+4139$ & $15: 50: 17.24$ & $+41: 39: 02.2$ & 0.22014 & Turn-on & {$[52468,57864]$} & XLT & This work \\
\hline $\mathrm{J} 1552+2737$ & $15: 52: 58.30$ & $+27: 37: 28.4$ & 0.08648 & Turn-on & {$[53498,56722]$} & LAMOST & This work \\
\hline J0126-0839 & $01: 26: 48.08$ & $-08: 39: 48.0$ & 0.19791 & Turn-off & {$[52163,54465]$} & SDSS & Ruan et al. (2016) \\
\hline $\mathrm{J} 0159+0033$ & 01:59:57.64 & $+00: 33: 10.5$ & 0.31204 & Turn-off & {$[51871,55201]$} & BOSS & LaMassa et al. (2015) \\
\hline $\mathrm{J} 1011+5442$ & 10:11:52.98 & $+54: 42: 06.4$ & 0.24639 & Turn-off & {$[52652,57073]$} & BOSS & Runnoe et al. (2016) \\
\hline $\mathrm{J} 1554+3629$ & $15: 54: 40.26$ & $+36: 29: 51.9$ & 0.23683 & Turn-on & {$[53172,57862]$} & XLT & Gezari et al. (2017) \\
\hline $\mathrm{J} 2336+0017$ & $23: 36: 02.98$ & $+00: 17: 28.7$ & 0.24283 & Turn-off & {$[52199,55449]$} & BOSS & Ruan et al. (2016) \\
\hline
\end{tabular}

Note. "Epoch" shows the MJD of the two epoch spectra. "Instrument2" indicates the spectrograph or telescope of the recent spectrum. XLT represents the Xinglong $2.16 \mathrm{~m}$ telescope at NAOC.

broad Balmer emission lines emerging (or enhancing), as well as increased broadband flux. The broad Balmer lines of $6 \mathrm{CL}$ AGNs disappeared (or faded), accompanied by dimming in the continuum. Among the $21 \mathrm{CL}$ AGNs, $17 \mathrm{CL}$ AGNs showed broad $\mathrm{H} \alpha, \mathrm{H} \beta, \mathrm{H} \gamma$, or even $\mathrm{H} \delta$ appeared or disappeared. Only broad $\mathrm{H} \alpha$ and $\mathrm{H} \beta$ changes were detected in $\mathrm{J} 0831+3646$, $\mathrm{J} 1319+6753$, and $\mathrm{J} 1552+2737$; clear emerging of broad $\mathrm{H} \alpha$ and $\mathrm{H} \beta$ emission lines, and increased flux in the optical and mid-infrared, confirmed their changes.

\subsection{AGN Optical and Mid-infrared Variability}

The continuum flux varies along with the CL AGN type transition. Table 7 summarizes the photometric variability of CL AGNs. We obtain the $g$-band variability, $\Delta g$, from the magnitude offset between the PS1 $g$-band magnitude, or the DELS $g$-band magnitude when available, and the SDSS $g$-band magnitude. We use the first epoch SDSS photometry if there are data at more than one epoch. The uncertainties of $\Delta g$ are 
Spectral Type Transition of CL AGNs

\begin{tabular}{|c|c|c|c|c|c|c|c|c|c|c|c|c|c|c|c|}
\hline Name & Redshift & $\begin{array}{c}\lambda L_{5100} \\
\left(10^{42} \mathrm{erg} \mathrm{s}^{-1}\right)\end{array}$ & $\mathrm{S} / \mathrm{N}_{1}$ & $\mathrm{~S} / \mathrm{N}_{2}$ & $\begin{array}{c}L_{\mathrm{H} \beta, 1} \\
\left(10^{41} \mathrm{erg} \mathrm{s}^{-1}\right)\end{array}$ & $\begin{array}{c}L_{\mathrm{H} \beta, 2} \\
\left(10^{41} \mathrm{erg} \mathrm{s}^{-1}\right)\end{array}$ & $\begin{array}{c}L_{\mathrm{H} \alpha, 1} \\
\left(10^{41} \mathrm{erg} \mathrm{s}^{-1}\right)\end{array}$ & $\begin{array}{c}L_{\mathrm{H} \alpha, 2} \\
\left(10^{41} \mathrm{erg} \mathrm{s}^{-1}\right)\end{array}$ & $\mathrm{S} / \mathrm{N}_{\mathrm{H} \beta_{1}}$ & $\mathrm{~S} / \mathrm{N}_{\mathrm{H} \beta_{2}}$ & $\mathrm{~S} / \mathrm{N}_{\mathrm{H} \alpha_{1}}$ & $\mathrm{~S} / \mathrm{N}_{\mathrm{H} \alpha_{2}}$ & E1 & E2 & Type \\
\hline $\mathrm{J} 0831+3646$ & 0.19501 & $18.23 \pm 0.24$ & 12.3 & 10.9 & $-4.05 \pm 0.33$ & $2.85 \pm 0.39$ & $3.60 \pm 1.00$ & $10.19 \pm 0.42$ & -12.1 & 7.3 & 3.6 & 24.2 & 2 & 1 & on \\
\hline $\mathrm{J} 0849+2747$ & 0.29854 & $63.42 \pm 1.48$ & 9.3 & 2.7 & $8.56 \pm 0.39$ & $-2.81 \pm 2.03$ & $22.76 \pm 1.95$ & $2.49 \pm 0.97$ & 22.2 & -1.4 & 11.7 & 2.6 & 1 & 2 & off \\
\hline J0909+4747 & 0.11694 & $6.97 \pm 0.20$ & 11.0 & 8.5 & $-1.88 \pm 0.13$ & $1.32 \pm 0.18$ & $1.48 \pm 0.13$ & $7.39 \pm 0.13$ & -14.9 & 7.5 & 11.8 & 57.1 & 1.9 & 1 & on \\
\hline $\mathrm{J} 0937+2602$ & 0.16219 & $6.85 \pm 0.32$ & 20.9 & 7.1 & $-1.71 \pm 0.13$ & $1.75 \pm 0.20$ & $3.66 \pm 0.24$ & $11.93 \pm 2.04$ & -12.8 & 8.6 & 15.4 & 5.9 & 1.9 & 1 & on \\
\hline $\mathrm{J} 1003+3525$ & 0.11886 & $45.77 \pm 1.64$ & 15.6 & 6.9 & $-1.40 \pm 0.12$ & $10.30 \pm 1.02$ & $2.34 \pm 0.57$ & $12.58 \pm 0.63$ & -11.7 & 10.1 & 4.1 & 19.9 & 2 & 1 & on \\
\hline $\mathrm{J} 1104+0118$ & 0.57514 & $302.41 \pm 6.30$ & 7.0 & 0.7 & $79.89 \pm 5.19$ & $-43.38 \pm 13.61$ & $\ldots$ & $\ldots$ & 15.4 & -3.2 & $\ldots$ & $\ldots$ & 1 & 2 & off \\
\hline $\mathrm{J} 1104+6343$ & 0.16427 & $4.46 \pm 0.49$ & 6.0 & 6.1 & $1.14 \pm 0.14$ & $0.33 \pm 0.11$ & $4.90 \pm 0.16$ & $0.79 \pm 0.13$ & 7.9 & 3.0 & 31.6 & 6.2 & 1 & 1.8 & off \\
\hline J1110-0003 & 0.21922 & $48.61 \pm 1.34$ & 8.0 & 6.1 & $0.27 \pm 0.10$ & $6.70 \pm 0.92$ & $1.78 \pm 0.71$ & $11.98 \pm 1.01$ & 2.8 & 7.3 & 2.5 & 11.9 & 2 & 1 & on \\
\hline $\mathrm{J} 1115+0544$ & 0.08995 & $17.27 \pm 0.27$ & 18.9 & 8.7 & $-1.23 \pm 0.07$ & $2.46 \pm 0.18$ & $0.05 \pm 0.07$ & $7.92 \pm 1.11$ & -18.4 & 14.0 & 0.7 & 7.2 & 2 & 1 & on \\
\hline $\mathrm{J} 1118+3203$ & 0.3651 & $56.48 \pm 2.29$ & 4.9 & 4.9 & $11.76 \pm 1.11$ & $2.90 \pm 0.51$ & $\ldots$ & $10.31 \pm 0.57$ & 10.6 & 5.7 & $\cdots$ & 18.0 & 1 & 1 & off $^{*}$ \\
\hline $\mathrm{J} 1132+0357$ & 0.09089 & $16.26 \pm 0.38$ & 17.3 & 7.4 & $-3.23 \pm 0.08$ & $4.74 \pm 0.40$ & $0.78 \pm 0.05$ & $2.40 \pm 0.17$ & -40.2 & 11.8 & 15.9 & 14.1 & 1.9 & 1 & on \\
\hline $\mathrm{J} 1150+3632$ & 0.34004 & $39.01 \pm 2.28$ & 4.9 & 5.2 & $5.02 \pm 0.53$ & $-3.29 \pm 0.49$ & $22.24 \pm 1.25$ & $3.18 \pm 0.40$ & 9.4 & -6.7 & 17.8 & 7.9 & 1 & 1.9 & off \\
\hline $\mathrm{J} 1152+3209$ & 0.37432 & $138.05 \pm 2.68$ & 11.9 & 3.7 & $38.31 \pm 1.16$ & $10.53 \pm 1.29$ & $\ldots$ & $\ldots$ & 33.0 & 8.2 & $\ldots$ & $\ldots$ & 1 & 1 & off $^{*}$ \\
\hline $\mathrm{J} 1259+5515$ & 0.19865 & $17.95 \pm 0.91$ & 7.4 & 3.5 & $0.02 \pm 0.24$ & $5.53 \pm 0.82$ & $4.30 \pm 0.38$ & $9.21 \pm 3.88$ & 0.1 & 6.8 & 11.4 & 2.4 & 1.9 & 1 & on \\
\hline $\mathrm{J} 1319+6753$ & 0.16643 & $30.90 \pm 1.46$ & 12.1 & 7.3 & $0.36 \pm 0.33$ & $7.96 \pm 1.16$ & $2.37 \pm 0.27$ & $5.90 \pm 4.43$ & 1.1 & 6.8 & 8.6 & 1.3 & 1.9 & 1 & on \\
\hline $\mathrm{J} 1358+4934$ & 0.11592 & $5.50 \pm 0.19$ & 7.8 & 13.1 & $0.36 \pm 0.09$ & $0.96 \pm 0.10$ & $0.74 \pm 0.06$ & $1.98 \pm 0.10$ & 3.8 & 9.2 & 13.4 & 19.0 & 1.9 & 1 & on \\
\hline $\mathrm{J} 1447+2833$ & 0.16344 & $66.68 \pm 0.97$ & 7.8 & 13.1 & $-1.16 \pm 0.29$ & $2.49 \pm 0.24$ & $2.27 \pm 0.19$ & $11.63 \pm 0.25$ & -3.9 & 10.6 & 12.2 & 47.2 & 1.9 & 1 & on \\
\hline $\mathrm{J} 1533+0110$ & 0.14268 & $<1.00$ & 14.2 & 13.4 & $-3.98 \pm 0.17$ & $0.54 \pm 0.12$ & $1.00 \pm 0.15$ & $3.97 \pm 0.29$ & -23.6 & 4.4 & 6.9 & 13.7 & 1.9 & 1.5 & on \\
\hline $\mathrm{J} 1545+2511$ & 0.11696 & $6.81 \pm 0.06$ & 19.6 & 17.1 & $-2.16 \pm 0.15$ & $0.71 \pm 0.11$ & $1.69 \pm 0.12$ & $5.90 \pm 0.14$ & -14.4 & 6.7 & 14.4 & 41.3 & 1.9 & 1 & on \\
\hline $\mathrm{J} 1550+4139$ & 0.22014 & $28.26 \pm 1.30$ & 11.6 & 8.1 & $-4.41 \pm 0.47$ & $15.71 \pm 1.48$ & $2.47 \pm 0.40$ & $14.01 \pm 0.89$ & -9.5 & 10.6 & 6.2 & 15.8 & 1.9 & 1 & on \\
\hline $\mathrm{J} 1552+2737$ & 0.08648 & $<1.00$ & 12.3 & 7.8 & $-3.67 \pm 0.05$ & $-1.34 \pm 0.10$ & $0.38 \pm 0.04$ & $2.70 \pm 0.05$ & -69.2 & -13.9 & 9.8 & 59.1 & 1.9 & 1.9 & on $^{*}$ \\
\hline
\end{tabular}

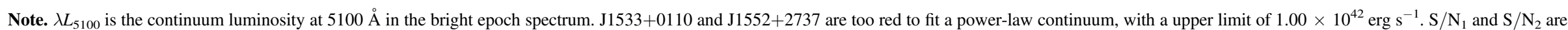

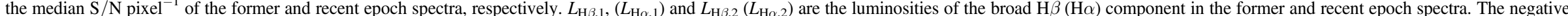

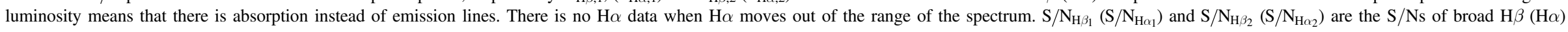
components in the former and recent epoch spectra. E1 and E2 describe the spectral types of the former and recent epoch spectra. Type describes the transition type. 
Table 7

Variability of Changing-look AGNs

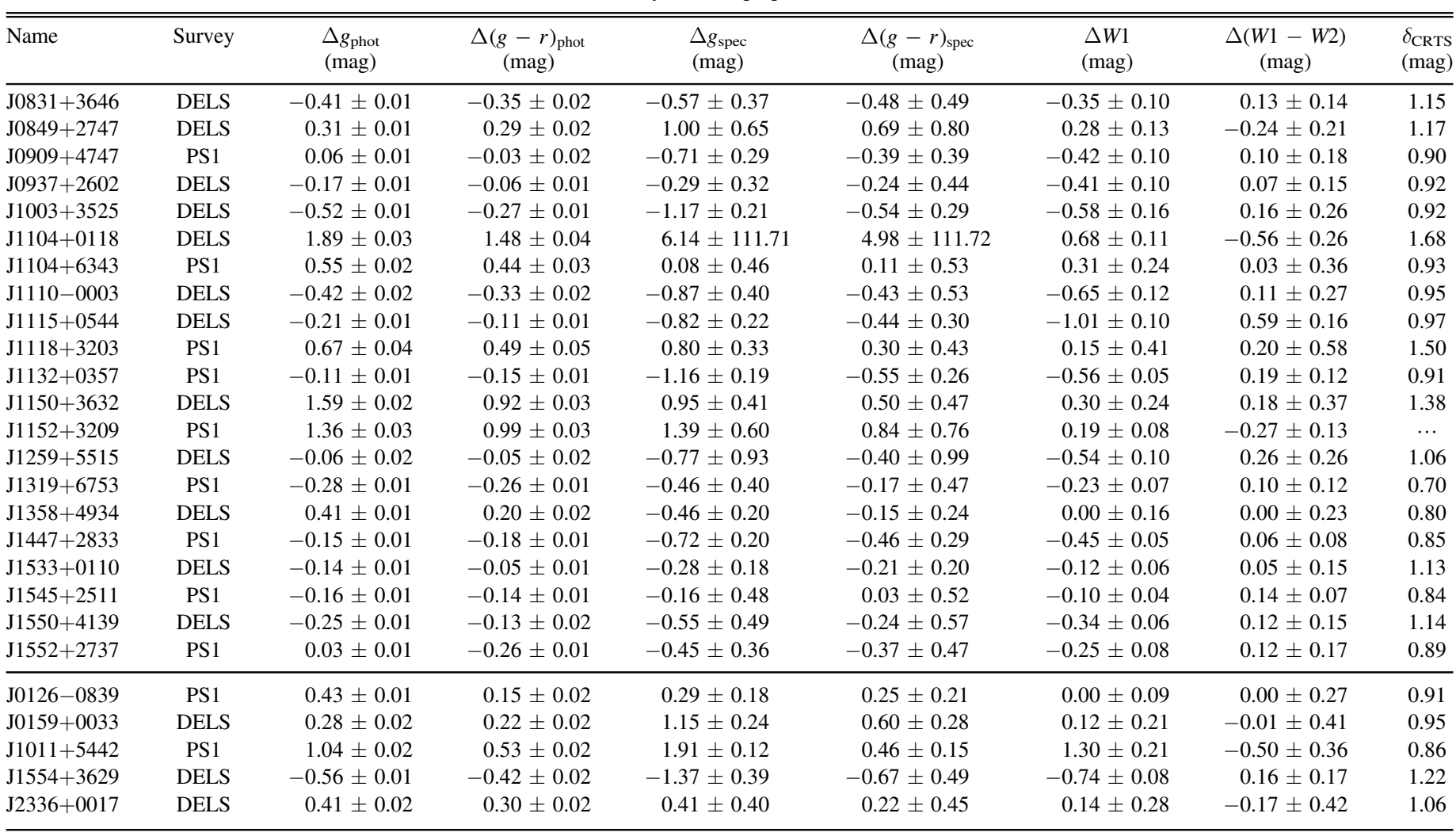

Note. $\Delta g_{\text {phot }}$ and $\Delta(g-r)_{\text {phot }}$ are the $g$-band variability and $g-r$ color variability from imaging data, while $\Delta g_{\text {spec }}$ and $\Delta(g-r)_{\text {spec }}$ are from spectrophotometry.

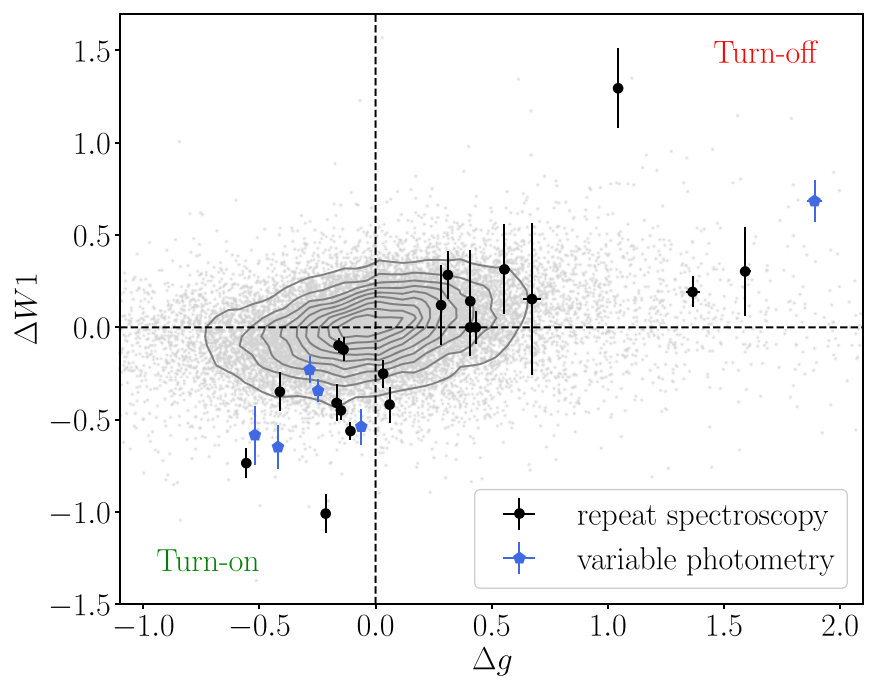

Figure 2. Variability of CL AGNs in the optical $g$-band and mid-infrared W1 band. The broadband fluxes in the optical and mid-infrared have the same trend. The CL AGNs marked as black dots are from repeat spectroscopy, and the blue pentagon shows CL AGNs selected from variable photometry. The region in the bottom left, where both the $g$ - and $W 1$-bands brighten, is a region for turn-on CL AGN selection; the top right area, where both $g$ and $W 1$ are dimmed, is useful for turn-off CL AGN selection. The gray dots and the contours shows quasars with a similar redshift range, $z<0.7$.

calculated from propagation of SDSS $g$-band magnitude uncertainty and PS1 $g$-band magnitude uncertainty, or the DELS $g$-band magnitude uncertainty when available. Similarly, the $g-r$ color variation, $\Delta(g-r)$, is calculated by the color offset between PS1 $g-r$ color, or the DELS $g-r$ color when available, and the SDSS $g-r$ color. The $g$-band variability ranges from -1.89 to $0.52 \mathrm{mag}$ in this sample. As in some CL AGN cases, the PS1 or DELS images were taken before the type transition. We also calculate the spectrophotometry with the spectra convolved with the SDSS filters. The spectrophotometry are calibrated by setting the first epoch SDSS spectrophotometry equal to the SDSS photometry at the closest epoch. The variations of spectrophotometry in the $g$-band and $g-r$ color are also listed in Table 7 . In the mid-infrared, there is a series of exposures within one day with a long interval of half a year in WISE multi-epoch data. We calculate the $3 \sigma$-clipped mean magnitude of WISE in $W 1$ and $W 2$ every half a year. The uncertainties of $W 1$ and $W 2$ are obtained from the $3 \sigma$-clipped standard deviation of $W 1$ and $W 2$ magnitude every half a year. There are less than 10 epochs of WISE mean magnitude for a single object. For the turn-on objects, the WISE variability is calculated from the offset between the brightest epoch photometry and the first epoch photometry. For turn-off objects, the WISE variability is calculated from the offset between the last epoch photometry and the brightest epoch photometry. The magnitude and color variability are calculated between the later epoch and the former epoch. Therefore, the magnitude variability is negative if an object becomes brighter, and the color variability is negative if the object becomes bluer. Figure 2 shows the optical broadband flux changes along with mid-infrared flux. In our sample, the mid-infrared variability $\Delta W 1$ ranges from -1.01 to 0.68 , and 15 of them varied for more than $0.2 \mathrm{mag}(|\Delta W 1|>0.2 \mathrm{mag})$. The mid-infrared flux is not significantly affected by dust extinction (Weingartner \& Draine 2001). In the scenario of variable obscuration, the variation in the $W 1$ band due to dust extinction yields a factor 

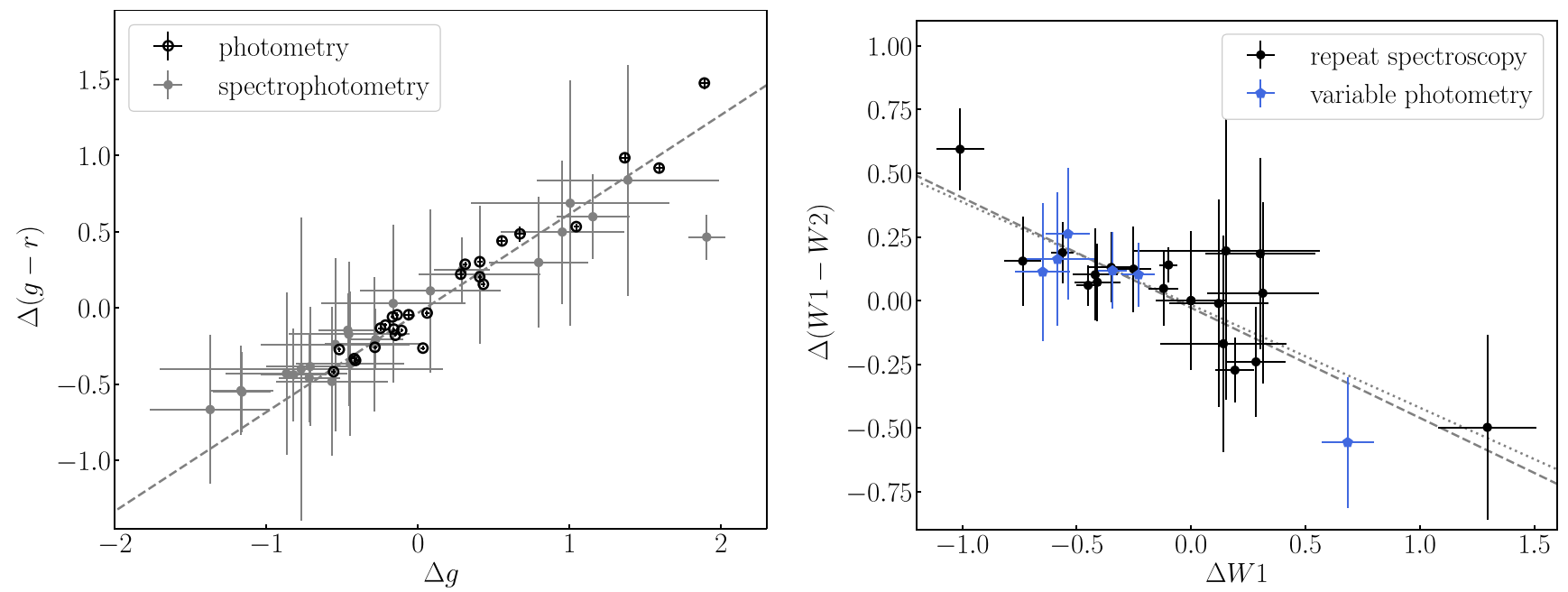

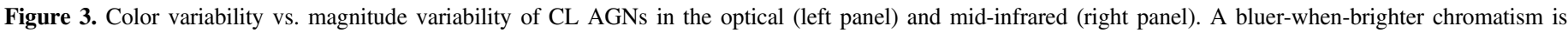

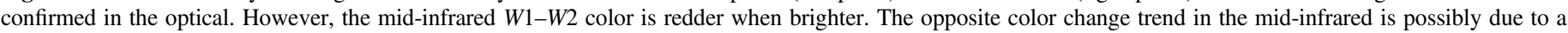
stronger contribution from the AGN dust torus when the AGN turns on.

of $\sim 21$ variability in $g$-band magnitude, according to the extinction curve in the optical and mid-infrared even considering micrometer-sized grains (Wang et al. 2015). A variability of 0.2 mag in the $W 1$ band suggests approximately 4.2 mag variability in the $g$-band. The optical variability, from photometric and spectrophotometric data, is not consistent with the scenario of variable obscuration in $10 \mathrm{CL}$ AGNs in our sample at more than the $3 \sigma$ confidence level $(\mathrm{J} 0831+3646$, $\mathrm{J} 0937+2602, \mathrm{~J} 1003+3525, \mathrm{~J} 1104+0118, \mathrm{~J} 1110-0003, \mathrm{~J} 1115$ $+0544, \mathrm{~J} 1132+0357, \mathrm{~J} 1259+5515, \mathrm{~J} 1447+2833$, and $\mathrm{J} 1550$ $+4139)$ and in eight CL AGNs between the $1 \sigma$ and $3 \sigma$ confidence levels (J0849+2747, J1104+6343, J1150+3632, $\mathrm{J} 1152+3209$ ，J1319+6753，J1533+0110，J1545+2511, and $\mathrm{J} 1552+2737$ ). The accuracy of the spectrophotometry is lower than that of the photometry. Following-up with high-accuracy photometric data after the type transition can better constrain the mechanism of type transition. Using mid-infrared variability to select CL AGNs, we were inclined to select CL AGNs with intrinsic changes instead of varying obscuration.

\section{Discussion}

\subsection{AGNs Color Variability}

The optical and mid-infrared colors vary following flux changes. Figure 3 shows the color variability versus the magnitude variability in the optical (left panel) and midinfrared (right panel). The bluer-when-brighter trend is a known trend in the optical (e.g., Wilhite et al. 2005; Schmidt et al. 2012; Zuo et al. 2012; Ruan et al. 2014). In the midinfrared, there is a trend in which $W 1-W 2$ is redder when brighter. A similar $W 1-W 2$ color dependence on AGN luminosity is reported in the Swift/Burst Alert Telescope AGN sample (Ichikawa et al. 2017).

The global SED variation helps to clarify the color changes in the infrared. We construct the infrared SED using the WISE data and the near-infrared data from the Two Micron All Sky Survey (2MASS; Skrutskie et al. 2006), which scanned the entire sky from 1997 to 2001 in three bands: $J(1.25 \mu \mathrm{m}), H$ $(1.65 \mu \mathrm{m})$, and $K_{s}(2.17 \mu \mathrm{m})$. Because there are no multi-epoch near-infrared and WISE W3 and W4 data, we show examples of two different objects in Figure 4. The two objects are J1115
+0544 (left panel) at the faint state before it turned on and $\mathrm{J} 0849+2747$ (right panel) before it turned off.

We fit the SED with the stellar and dust torus emission components. The simple stellar synthesis model by Bruzual \& Charlot (2003, hereafter BC03) with an age of 5 Gyr and solar metallicity is used for the stellar emission. The dust torus component is described by a new version of the radiative transfer model CAT3D (Hönig \& Kishimoto 2017). Due to limitations of the data, we adopt the templates without the wind component. We perform the SED fitting with a new Markov chain Monte Carlo (MCMC) method (Shangguan et al. 2018) combining the stellar and dust torus models.

In the SED before the AGN turned on, the $W 1$ and $W 2$ bands generally follow the stellar emission. In the SED after the AGN turned on, the $W 1$ and $W 2$ bands, especially $W 2$, were strongly affected by the dust torus radiation. Therefore, it is possible that the $W 1-W 2$ shows as blue without a strong AGN contribution, and turns out to be red after the AGN turning on. Thus the midinfrared $W 1-W 2$ color of CL AGNs turns to be redder when brighter.

In the optical, the color-magnitude variation relation (dashed line in left panel in Figure 3) of CL AGNs (listed in Table 5) is fitted by a least-squares fitting algorithm as

$$
\Delta(g-r)=(0.650 \pm 0.042) \Delta g-(0.034 \pm 0.017) .
$$

The CL AGNs selected from repeat spectroscopy are not biased by WISE variability selection criteria. For CL AGNs selected from repeat spectroscopy, the mid-infrared colormagnitude relation (dotted line in left panel in Figure 3) is fitted as

$$
\begin{aligned}
& \Delta(W 1-W 2) \\
& \quad=(-0.404 \pm 0.078) \Delta W 1-(0.016 \pm 0.033) .
\end{aligned}
$$

Thus, a selection criterion based on mid-infrared variability as $|\Delta(W 1-W 2)|>0.1$ when $|\Delta W 1|>0.2$, is reasonable. With all the CL AGNs (in Table 5), the color-magnitude relation in the mid-infrared (dashed line in the left panel in Figure 3 ) is fitted as

$$
\begin{aligned}
& \Delta(W 1-W 2) \\
& \quad=(-0.432 \pm 0.066) \Delta W 1-(0.028 \pm 0.028) .
\end{aligned}
$$



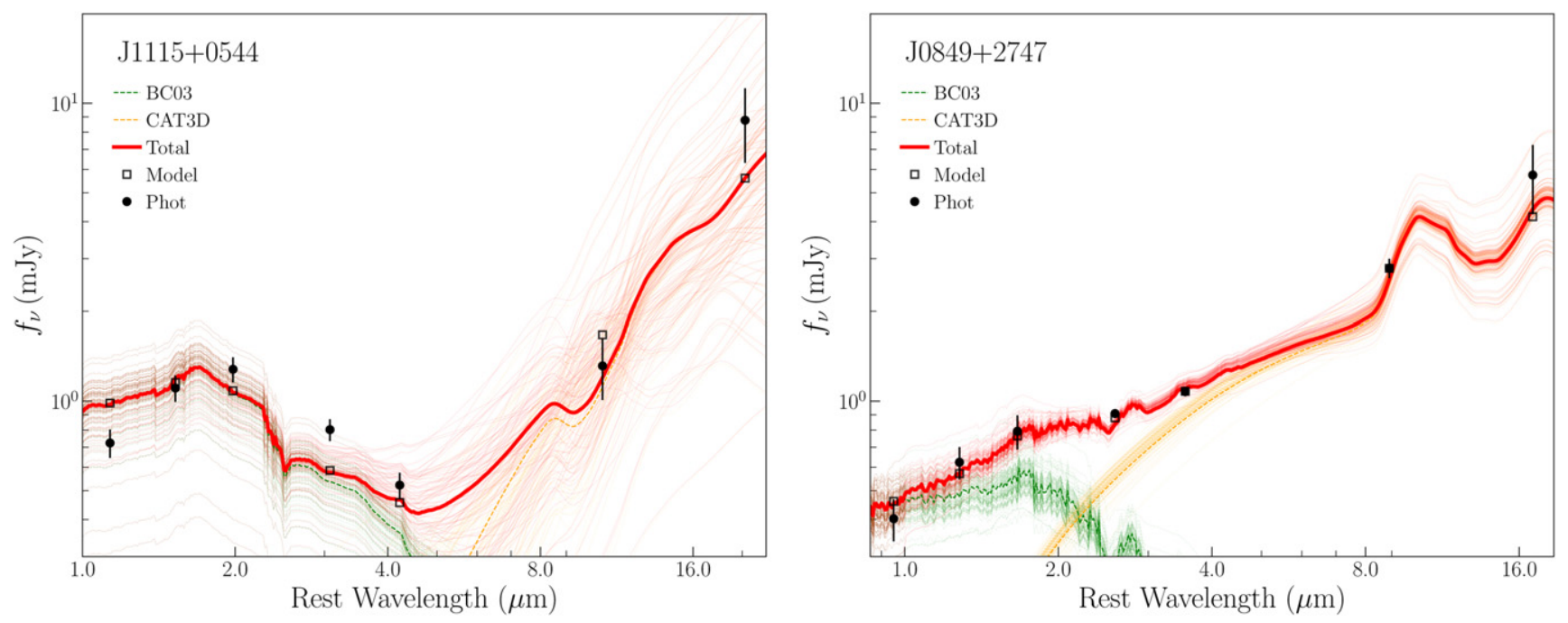

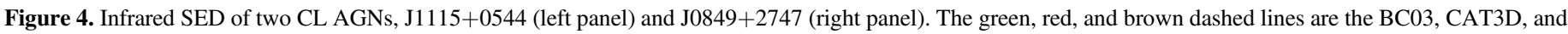

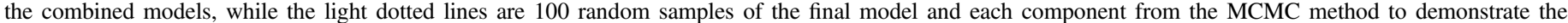

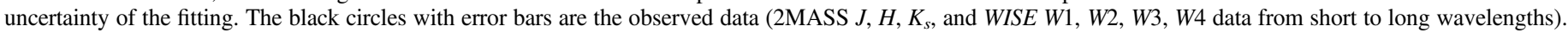
The empty squares are the modeled photometric data at the observed bands.

The CL AGNs, selected from WISE variability, obey the colormagnitude relation well. The mid-infrared variability and color variability are good tracers for CL AGNs with intrinsic changes.

\subsection{The Timescale of the Type Transition}

The current data coverage is not good enough to measure the timescale of transition. Therefore, we only roughly estimate the upper limit of type transition as follows. The upper limit of the transition timescale is obtained by the time interval between the former and recent spectroscopic epoch $\left(\Delta t_{\text {spec }}\right.$ in the restframe in Table 8). We further prescribe a limit to the timescale with the light curve changes approaching the transition. For example, although the two spectra of $\mathrm{J} 1115+0544$ were separated by 14 years, there was no variability detected in SDSS, PS1, and CRTS imaging data. The rapid flux brightening was detected by WISE in 2015. The W1 of J1115+0544 increased for more than $1 \mathrm{mag}$ in a short interval, less than one year in the rest-frame. The light curves of CL AGNs keep quiescent at faint states, namely before the transition of turn-on CL AGNs or after the transition of turn-off CL AGNs. The CL AGNs usually vary in their AGN phase. In some cases (for example, J0831+3646, J1110-0003, J1115+0544, J1259 +5515 , and $\mathrm{J} 1319+6753)$, the WISE flux increased when the AGN turned on, and decreased with a smaller amplitude later on, possibly due to the variability of the AGN accretion rate. On the other hand, it is also possible that the flux increase is due to the TDEs instead of AGN activity. In this scenario, the later light curve should continually decrease following the typical light curve of TDEs, which is proportional to $t^{-5 / 3}$ (Rees 1988; Lodato et al. 2009; Guillochon \& Ramirez-Ruiz 2013). Follow-up photometric data are needed to distinguish the scenarios of AGN accretion rate variability and TDEs. The epoch approaching the type transition is obtained by a recent quiescent image epoch at the faint state, or the brightest image epoch in their AGN phase. In Table 8, Epoch1* and Epoch2* show the imaging data epochs (and imaging survey) approaching the transition before and after the type transition.
$\Delta t$, obtained from photometric variation approaching the type transition when available, is a better upper limit of the transition timescale in the rest-frame than $\Delta t_{\text {spec }}$. Therefore, the upper limit of the type transition timescales ranges from 0.9 to 12.6 years in the rest-frame.

The short transition timescale is not consistent with the scenario of variable obscuration (e.g., LaMassa et al. 2015; Gezari et al. 2017; Sheng et al. 2017). In a scenario of variable accretion rate, the CL timescale in this sample is much shorter than the inflow timescale of gas in the inner parts of the accretion disk, as discussed in previous works (LaMassa et al. 2015; MacLeod et al. 2016; Runnoe et al. 2016; Gezari et al. 2017). So modeling CL AGNs via changes in accretion rates is far from a settled matter. A detailed report on the mechanisms of the type transition will be presented in a subsequent paper.

\section{Summary}

We present surveys of CL AGNs in the SDSS spectra archive, the LAMOST spectra archive, and observations for some CL AGN candidates selected from photometric data. In total, we discover 21 new CL AGNs at $0.08<z<0.58$. Among the new CL AGNs, 5 were found by repeat spectra from the SDSS, 10 were discovered from repeat spectra in SDSS and LAMOST, and 6 were selected from photometric variability and confirmed by new spectroscopy. From our surveys, approximately $0.006 \%(0.007 \%)$ galaxies with repeat spectroscopy in the SDSS (SDSS and LAMOST) are CL AGNs, with obvious broad Balmer emission-line changes.

The physical mechanism of type transition is important for understanding the evolution of AGNs. The mid-infrared flux changes with the optical continuum flux. Variations of more than 0.2 mag in the mid-infrared were detected in $15 \mathrm{CL}$ AGNs during the transition, and such variability suggests $\sim 4.2 \mathrm{mag}$ variability in the $g$-band. In the scenario of variable obscuration, the variation in the $W 1$ band due to dust extinction yields a factor of $\sim 21$ variability in the $g$-band. The optical variability is not consistent with the scenario of variable obscuration in the 
Table 8

Timescale of Changing-look AGN Transition

\begin{tabular}{|c|c|c|c|c|c|c|c|}
\hline Name & Redshift & Epoch1 & Epoch2 & $\begin{array}{c}\Delta t_{\text {spec }} \\
\text { (years) }\end{array}$ & Epoch $1^{*}$ & Epoch2* & $\begin{array}{c}\Delta t \\
\text { (years) }\end{array}$ \\
\hline $\mathrm{J} 0831+3646$ & 0.19501 & 52312 & 57367 & 11.6 & PS1 56244 & $\ldots$ & 2.6 \\
\hline $\mathrm{J} 0849+2747$ & 0.29854 & 53350 & 56628 & 6.9 & $\ldots$ & $\ldots$ & 6.9 \\
\hline J0909+4747 & 0.11694 & 52620 & 57745 & 12.6 & $\cdots$ & $\ldots$ & 12.6 \\
\hline $\mathrm{J} 0937+2602$ & 0.16219 & 54524 & 57369 & 6.7 & PS1 55845 & $\ldots$ & 3.6 \\
\hline $\mathrm{J} 1003+3525$ & 0.11886 & 53389 & 57867 & 11.0 & PS1 55726 & $\ldots$ & 5.2 \\
\hline $\mathrm{J} 1104+0118$ & 0.57514 & 52374 & 57867 & 9.6 & $\ldots$ & PS1 55902 & 6.1 \\
\hline $\mathrm{J} 1104+6343$ & 0.16427 & 52370 & 54498 & 5.0 & $\ldots$ & $\ldots$ & 5.0 \\
\hline $\mathrm{J} 1110-0003$ & 0.21922 & 51984 & 57864 & 13.2 & & WISE 57003 & 11.3 \\
\hline $\mathrm{J} 1115+0544$ & 0.08995 & 52326 & 57393 & 12.7 & WISE 57002 & WISE 57367 & 0.9 \\
\hline $\mathrm{J} 1118+3203$ & 0.3651 & 53431 & 56367 & 5.9 & $\ldots$ & $\ldots$ & 5.9 \\
\hline $\mathrm{J} 1132+0357$ & 0.09089 & 52642 & 57392 & 11.9 & CRTS 56665 & $\ldots$ & 1.8 \\
\hline $\mathrm{J} 1150+3632$ & 0.34004 & 53436 & 57422 & 8.1 & $\ldots$ & $\ldots$ & 8.1 \\
\hline $\mathrm{J} 1152+3209$ & 0.37432 & 53446 & 57844 & 8.8 & $\ldots$ & PS1 56063 & 5.2 \\
\hline $\mathrm{J} 1259+5515$ & 0.19865 & 52707 & 57863 & 11.8 & WISE 55534 & WISE 56806 & 2.9 \\
\hline $\mathrm{J} 1319+6753$ & 0.16643 & 51988 & 57867 & 13.8 & $\ldots$ & WISE 56982 & 11.7 \\
\hline $\mathrm{J} 1358+4934$ & 0.11592 & 53438 & 54553 & 2.7 & $\ldots$ & $\ldots$ & 2.7 \\
\hline $\mathrm{J} 1447+2833$ & 0.16344 & 53764 & 57071 & 7.8 & $\cdots$ & $\ldots$ & 7.8 \\
\hline $\mathrm{J} 1533+0110$ & 0.14268 & 51989 & 54561 & 6.2 & $\cdots$ & $\cdots$ & 6.2 \\
\hline $\mathrm{J} 1545+2511$ & 0.11696 & 53846 & 57891 & 9.9 & $\ldots$ & $\cdots$ & 9.9 \\
\hline $\mathrm{J} 1550+4139$ & 0.22014 & 52468 & 57864 & 12.1 & PS1 56233 & $\ldots$ & 3.7 \\
\hline $\mathrm{J} 1552+2737$ & 0.08648 & 53498 & 56722 & 8.1 & PS1 55987 & $\cdots$ & 1.9 \\
\hline
\end{tabular}

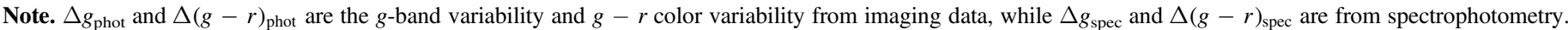

$10 \mathrm{CL}$ AGNs in our sample at more than the $3 \sigma$ confidence level and in $8 \mathrm{CL}$ AGNs between the $1 \sigma$ and $3 \sigma$ confidence levels. Follow-up high-accuracy photometric data after the type transition can better constrain the mechanism of the type transition.

The optical and mid-infrared colors change with flux variation. A bluer-when-brighter chromatism is confirmed in the optical bands. However, the mid-infrared $W 1-W 2$ color is redder when brighter. The opposite color change trend in the mid-infrared is possibly caused by a stronger contribution from the AGN dust torus when the AGNs turn on. The mid-infrared variability and colors are good tracers for CL AGNs with intrinsic variability.

The upper limit of type transition timescales ranges from 0.9 to 12.6 years in the rest-frame. The mid-infrared emission of $\mathrm{J} 1115+0544$ increased for more than $1 \mathrm{mag}$ in a short interval, less than one year in the rest-frame. The timescale of the type transition will help distinguish the mechanism of the changes. Follow-up photometric data are needed to distinguish the scenarios of AGN accretion rate variability and TDEs based on the decreasing light curve trend of TDEs $\left(\sim t^{-5 / 3}\right)$.

The photometric variability of CL AGNs provides ways to select CL AGNs from large-area surveys. In the future, the Large Synoptic Survey Telescope (LSST; Ivezic et al. 2008), with multi-epoch and multi-band data, will provide powerful data for CL AGN selection and monitoring.

In future papers we will analyze the spectra and imaging data in more detail, and discuss their probable transition mechanism. The rareness of CL AGNs provides exceptional opportunities studies of the $M-\sigma_{*}$ relation at higher redshift with faint-state spectra and AGN-phase spectra. We plan to estimate their black hole masses from the $M-\sigma_{*}$ relation, and compare with their virial black hole masses obtained from the single-epoch spectra. The images of CL AGNs at faint states are useful for studies of AGN host galaxies and help to avoid contamination from the luminous central engines. We will perform a statistical study on the host galaxy properties of CL AGNs.

The work is supported by the the National Key R\&D Program of China (2016YFA0400703), National Key Basic Research Program of China 2014CB845700, the NSFC grant No.11373008 and No.11533001. We thank Luis Ho, Yue Shen, Arjun Dey, Nicholas Ross, Aaron Meisner, and Ning Jiang, Yanxia Xie for very helpful discussions. We thank Shu Wang for providing the extinction data.

We acknowledge the use of SDSS data. Funding for SDSSIII has been provided by the Alfred P. Sloan Foundation, the Participating Institutions, the National Science Foundation, and the U.S. Department of Energy Office of Science. The SDSSIII website is http://www.sdss3.org/. SDSS-III is managed by the Astrophysical Research Consortium for the Participating Institutions of the SDSS-III Collaboration including the University of Arizona, the Brazilian Participation Group, Brookhaven National Laboratory, Carnegie Mellon University, University of Florida, the French Participation Group, the German Participation Group, Harvard University, the Instituto de Astrofisica de Canarias, the Michigan State/Notre Dame/ JINA Participation Group, Johns Hopkins University, Lawrence Berkeley National Laboratory, Max Planck Institute for Astrophysics, Max Planck Institute for Extraterrestrial Physics, New Mexico State University, New York University, Ohio State University, Pennsylvania State University, University of Portsmouth, Princeton University, the Spanish Participation Group, University of Tokyo, University of Utah, Vanderbilt University, University of Virginia, University of Washington, and Yale University.

We acknowledge the use of LAMOST data. The Large Sky Area Multi-Object Fiber Spectroscopic Telescope (LAMOST, also named Guoshoujing Telescope) is a National Major Scientific Project built by the Chinese Academy of Sciences. Funding for the project has been provided by the National 

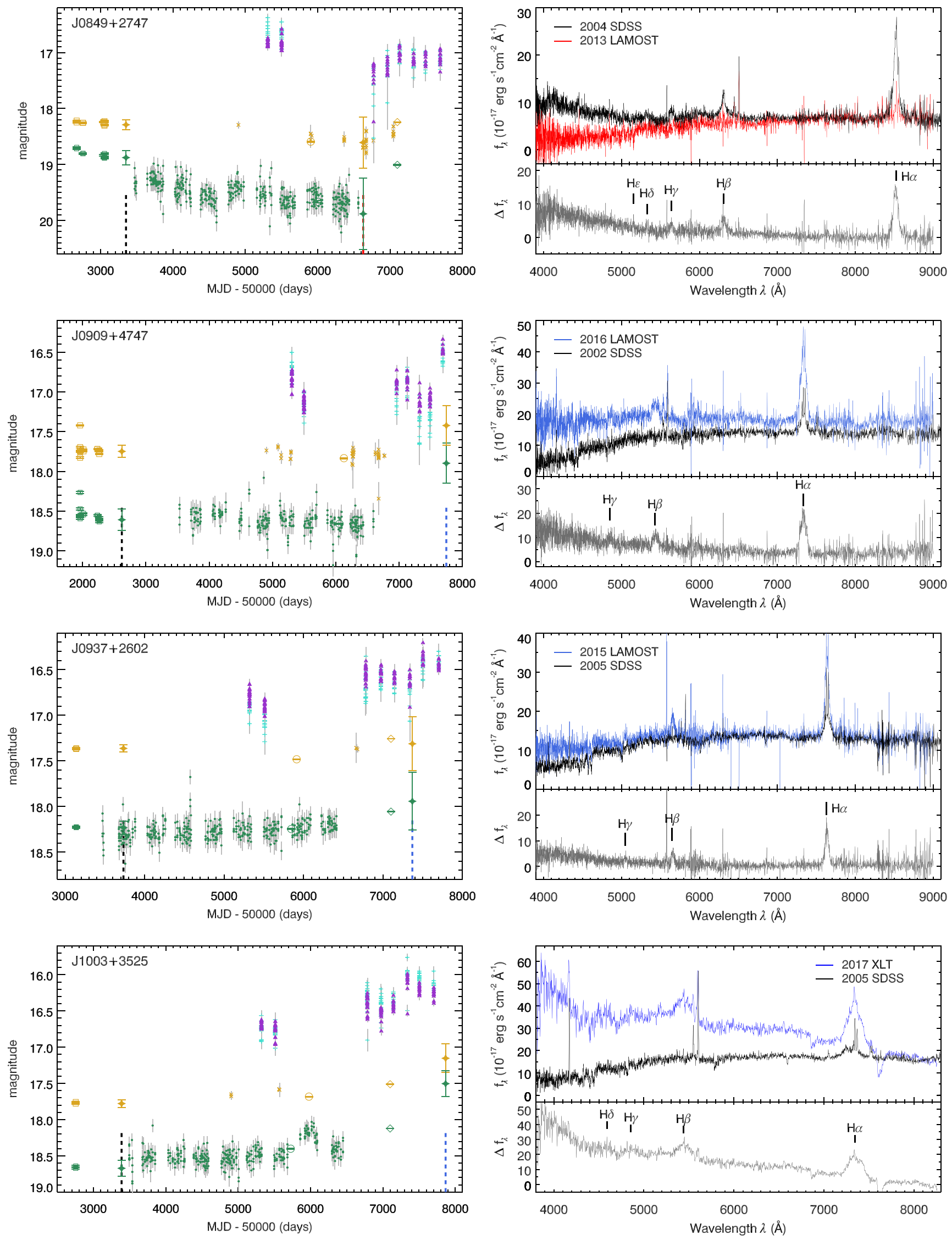

Figure 5. Same as Figure 1. 

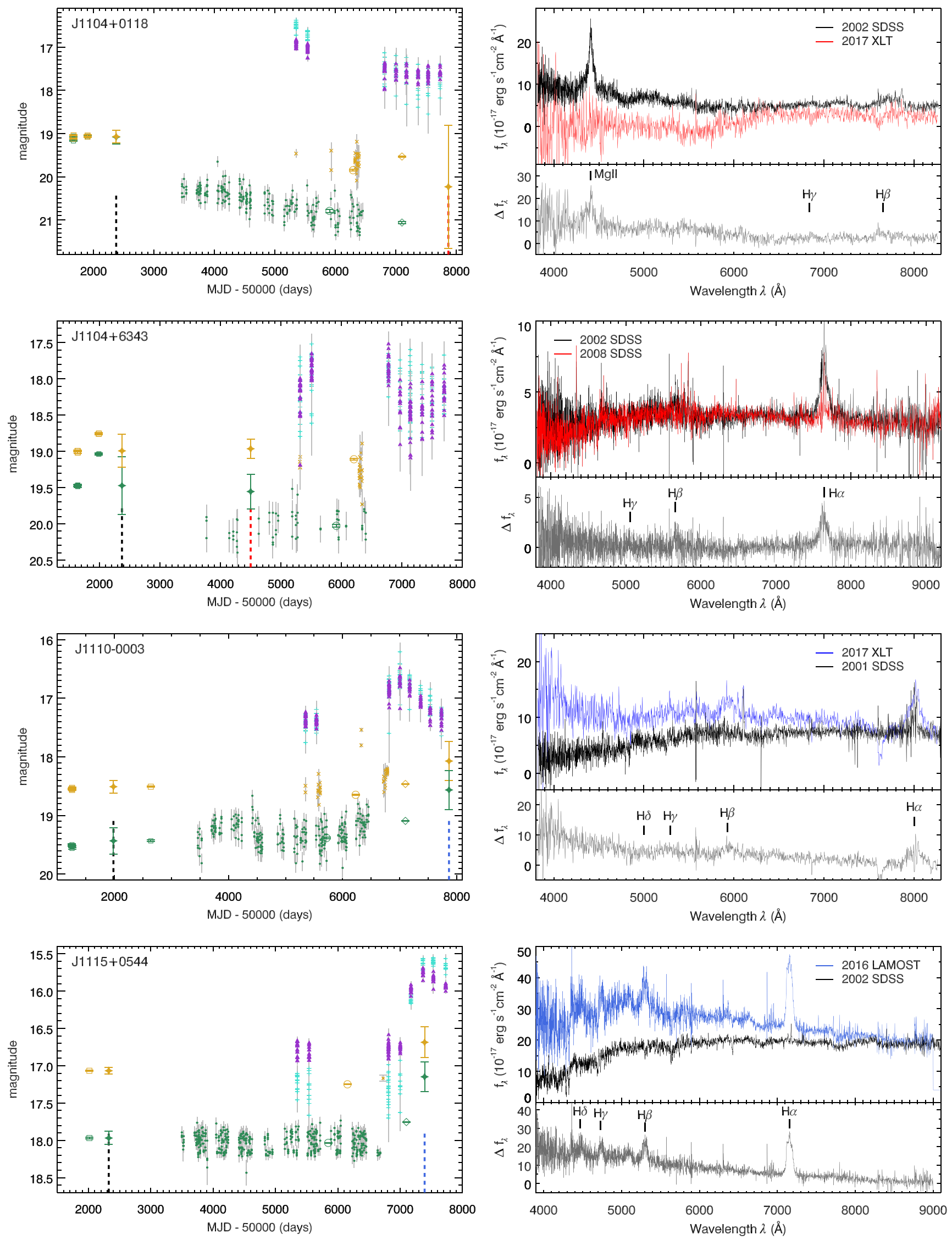

Figure 5. (Continued.) 

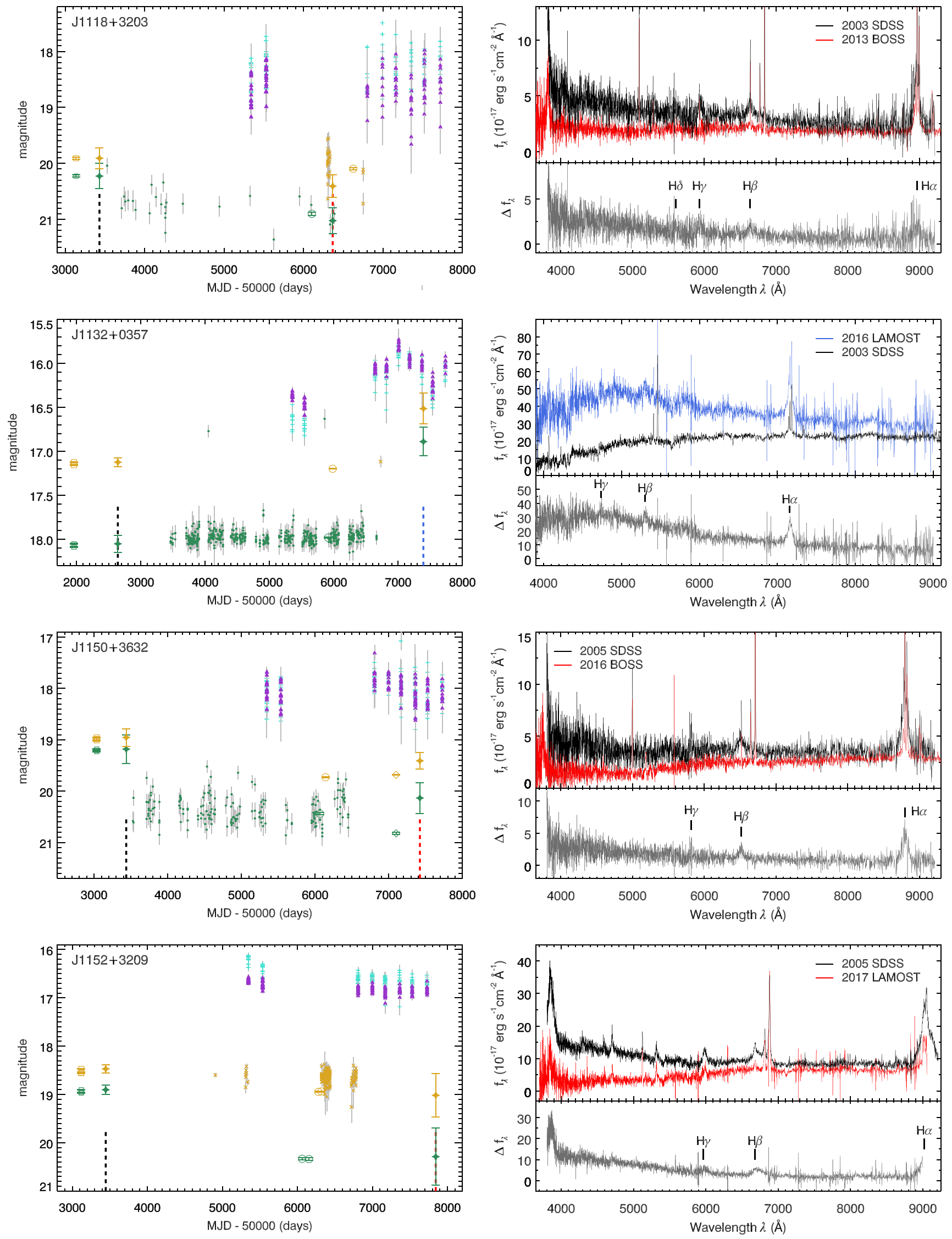

Figure 5. (Continued.) 

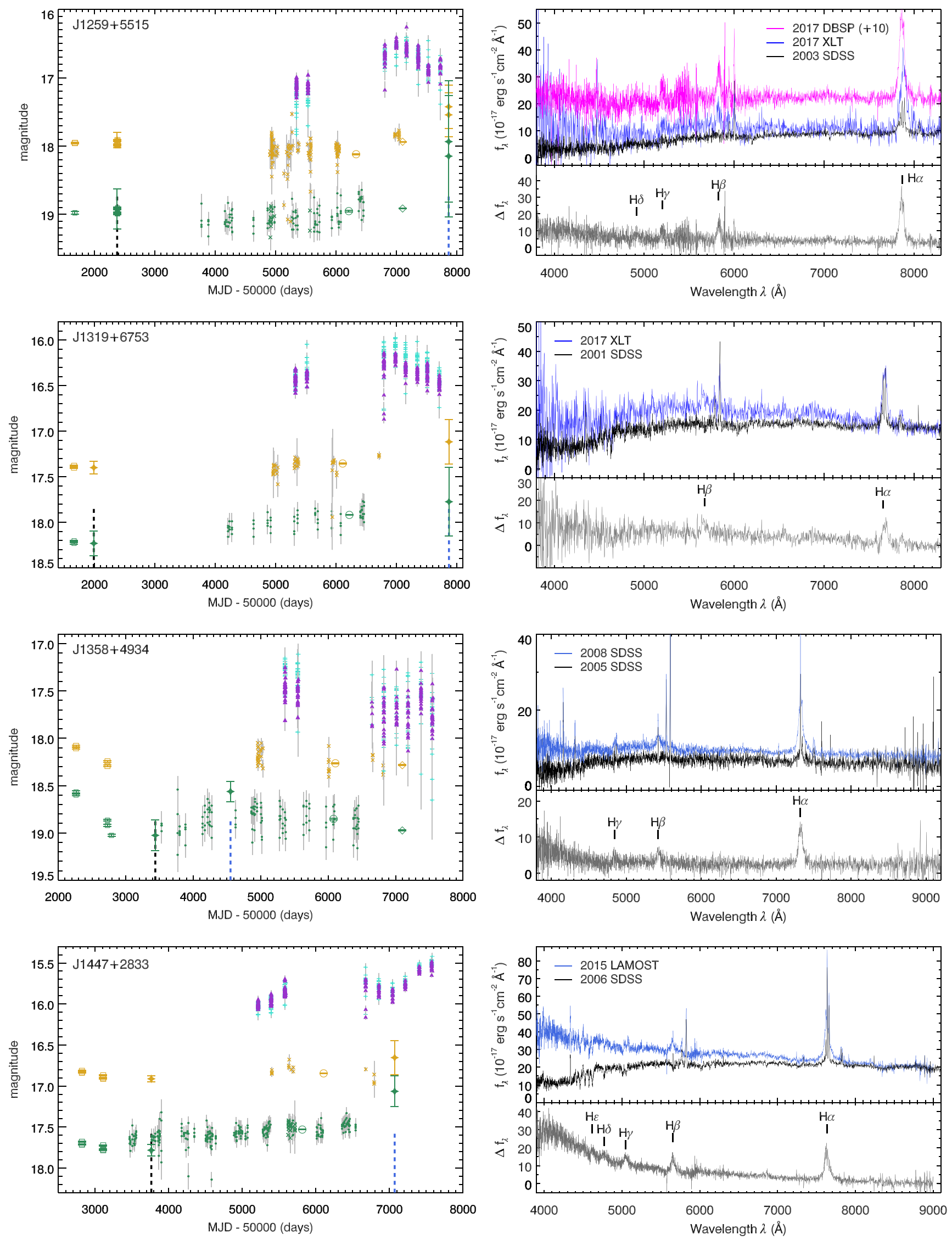

Figure 5. (Continued.) 

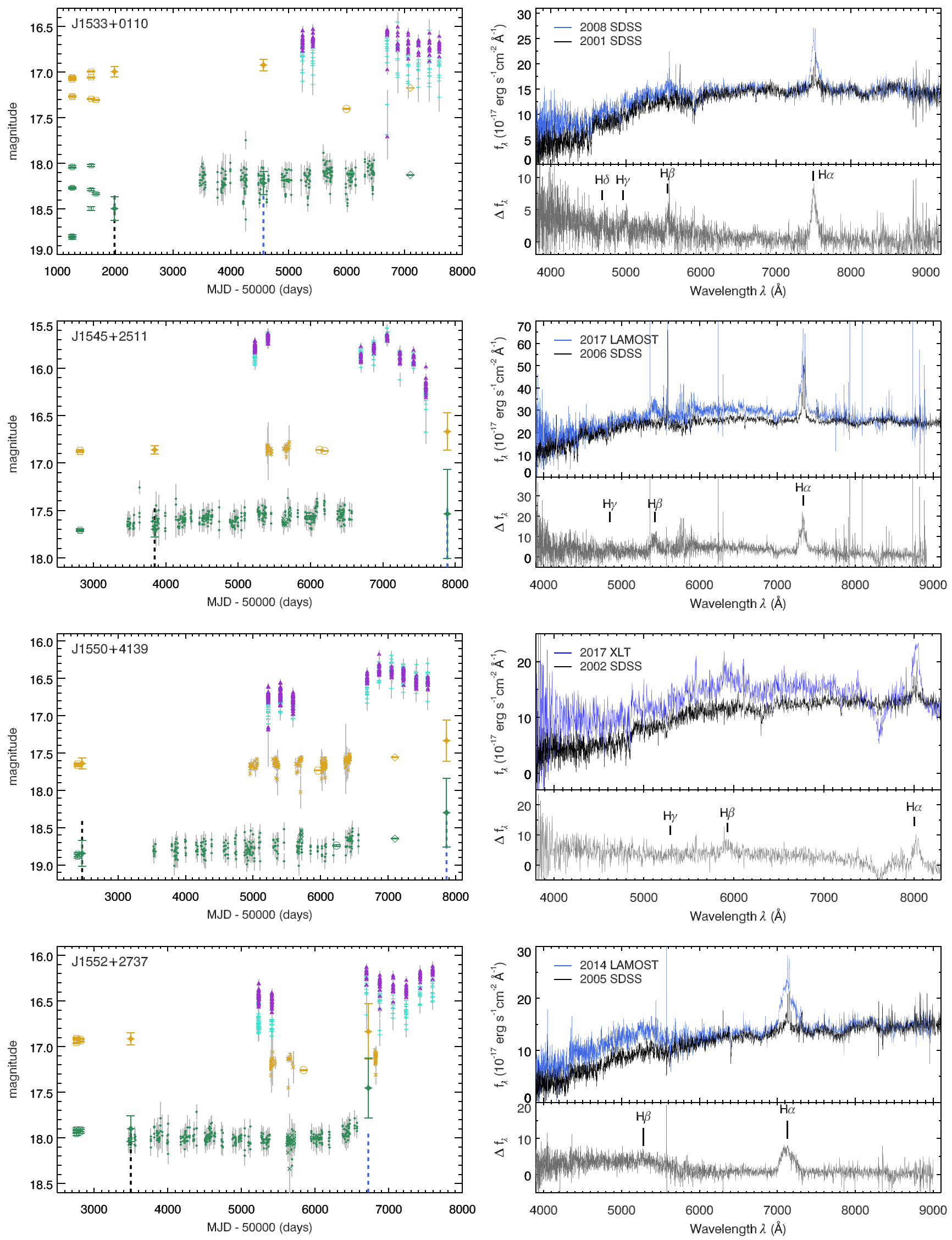

Figure 5. (Continued.) 

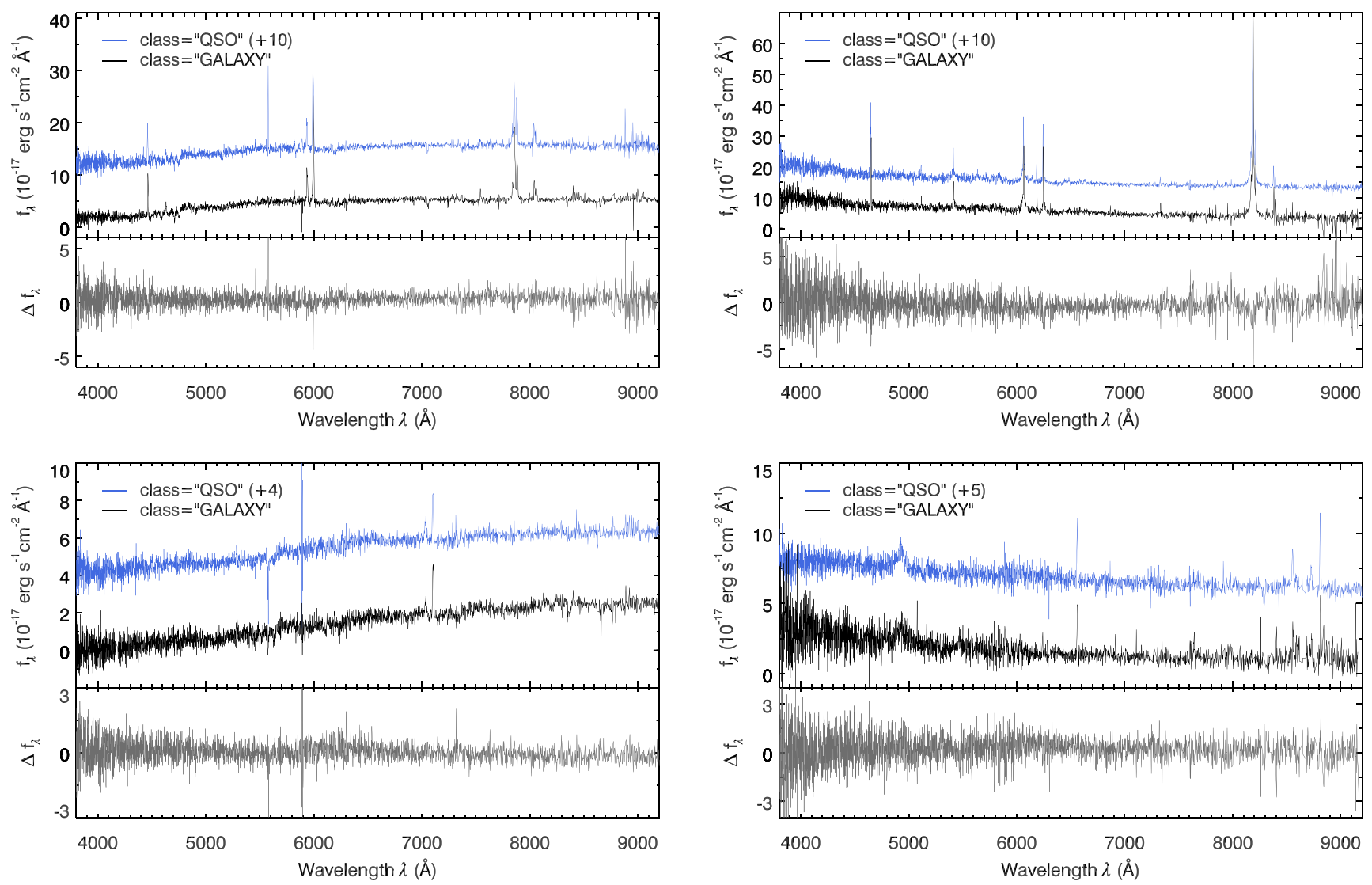

Figure 6. Four CL candidates in the SDSS that were rejected by visual inspection. From low to high redshift, the four objects are J0001-0005 (left upper panel), $\mathrm{J} 1356-0115$ (right upper panel), J0803+4258 (left bottom panel), and J0937+3232 (right bottom panel). To show the spectra at different epochs, a constant is added to the spectra that were classified as "QSO."

Development and Reform Commission. LAMOST is operated and managed by the National Astronomical Observatories, Chinese Academy of Sciences.

This research has made use of PS1, DELS, WISE, CRTS, and PTF imaging data. The PS1 has been made possible through contributions by the Institute for Astronomy, the University of Hawaii, the Pan-STARRS Project Office, the Max-Planck Society and its participating institutes, the Max Planck Institute for Astronomy, Heidelberg and the Max Planck Institute for Extraterrestrial Physics, Garching, The Johns Hopkins University, Durham University, the University of Edinburgh, Queen's University Belfast, the HarvardSmithsonian Center for Astrophysics, the Las Cumbres Observatory Global Telescope Network Incorporated, the National Central University of Taiwan, the Space Telescope Science Institute, the National Aeronautics and Space Administration under grant No. NNX08AR22G issued through the Planetary Science Division of the NASA Science Mission Directorate, the National Science Foundation under grant No. AST-1238877, the University of Maryland, and Eotvos Lorand University (ELTE). We acknowledge the use of the DESI Legacy imaging survey, whose website is http://legacysurvey. org. This research has made use of the NASA/ IPAC Infrared Science Archive, which is operated by the Jet Propulsion Laboratory, California Institute of Technology, under contract with the National Aeronautics and Space Administration. This publication makes use of data products from the Wide-field Infrared Survey Explorer, which is a joint project of the University of California, Los Angeles, and the Jet Propulsion
Laboratory/California Institute of Technology, funded by the National Aeronautics and Space Administration. The CSS survey is funded by the National Aeronautics and Space Administration under grant No. NNG05GF22G issued through the Science Mission Directorate Near-Earth Objects Observations Programme. The CRTS survey is supported by the US National Science Foundation under grants AST-0909182 and AST-1313422. We acknowledge the use of PTF data; the PTF website is https://www.ptf.caltech.edu.

We acknowledge the use of the Xinglong $2.16 \mathrm{~m}$ telescope and the Palomar Hale $5 \mathrm{~m}$ telescope. This work has made use of the TOPCAT (Taylor 2005). We thank the Chinese Virtual Observatory; its website is http://www.china-vo.org.

Facilities: Sloan, PS1, IRSA, WISE, Beijing: $2.16 \mathrm{~m}$ (BFOSC), Palomar P200/Caltech.

\section{Appendix}

The light curves and spectra of the other 20 new CL AGNs, in addition to the CL AGN shown in Figure 1, are provided in Figure 5.

Figure 6 shows some examples of CL candidates in the SDSS rejected by our visual inspection. Table 9 summarizes some details about the four CL candidates. They were were classified as "QSO" and "GALAXY" at different epochs in the SDSS. As shown in the residual spectra, there is no dramatic change. As the SDSS spectroscopic pipelines classify the objects through a comparison of individual spectra with galaxy, QSO, and stellar templates, these objects were classified as 
Table 9

Examples of CL Candidates Rejected by Visual Inspection in the SDSS

\begin{tabular}{lccccc}
\hline \hline Name & R.A. & Decl. & Redshift & Epoch(GALAXY) & Epoch(QSO) \\
\hline J0001-0005 & $00: 01: 07.52$ & $-00: 05: 52.3$ & 0.19754 & 55477 & 51942 \\
J1356-0115 & $13: 56: 18.49$ & $-01: 15: 14.0$ & 0.24726 & 55245 & 52721 \\
J0803+4258 & $08: 03: 47.55$ & $+42: 58: 38.9$ & 0.41881 & 54807 & 55178 \\
J0937+3232 & $09: 37: 35.46$ & $+32: 32: 49.9$ & 0.76017 & 56310 \\
\hline
\end{tabular}

Note. Epoch(GALAXY) and Epoch(QSO) describe the MJDs of the spectra that were classified as "GALAXY" and "QSO," respectively.

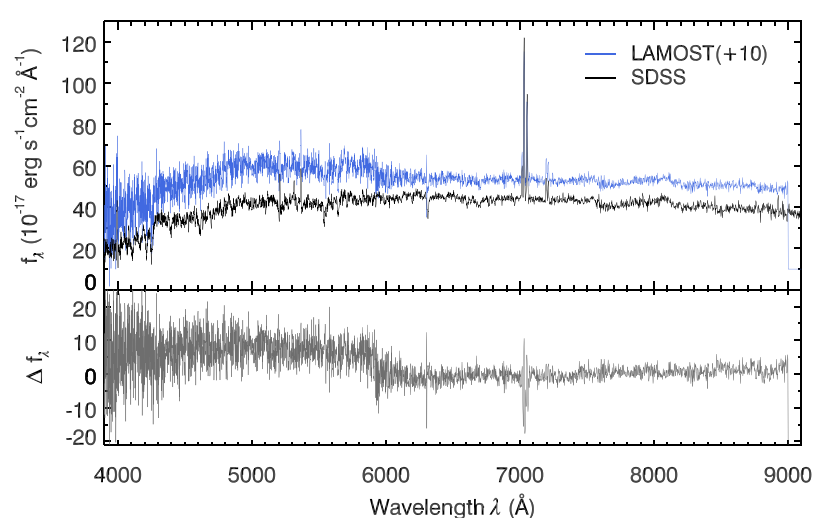

Figure 7. Example of CL candidates in SDSS and LAMOST that were rejected by visual inspection.

different types possibly due to marginally different spectral $\mathrm{S} / \mathrm{Ns}$ at different epochs.

The spectra were classified by the LAMOST 1D pipeline into four primary classifications, namely "STAR," "GALAXY," "QSO," and "UNKNOWN," through matching individual spectra with templates (Luo et al. 2015). Among the 8171 objects, approximately $36 \%$ of them are classified as "UNKNOWN" and 7\% are classified as "STAR." The S/N of objects classified as "UNKNOWN" is low due to unstable fiber efficiency, non-photometric observational conditions, or they are too faint for the LAMOST survey (Ai et al. 2016). None of the $10 \mathrm{CL}$ AGNs from LAMOST were classified as "UNKNOWN" or "STAT." Figure 7 shows an example of CL candidates in the SDSS and LAMOST that were rejected by visual inspection. There is a break around $5700-5900 \AA$ in the LAMOST spectrum due to inappropriate combination of the spectra in the blue and red arms, resulting in flux intensity variation in the automatic program check process.

\section{ORCID iDs}

Qian Yang (i) https://orcid.org/0000-0002-6893-3742 Xue-Bing Wu 10 https://orcid.org/0000-0002-7350-6913 Xiaohui Fan (10) https://orcid.org/0000-0003-3310-0131 Linhua Jiang (i) https://orcid.org/0000-0003-4176-6486 Ian McGreer (1) https://orcid.org/0000-0002-3461-5228 Jinyi Shangguan (i) https://orcid.org/0000-0002-4569-9009 Feige Wang (1) https://orcid.org/0000-0002-7633-431X Jinyi Yang (1) https://orcid.org/0000-0001-5287-4242

\section{References}

Abazajian, K. N., Adelman-McCarthy, J. K., Agüeros, M. A., et al. 2009, ApJS, 182, 543

Abolfathi, B., Aguado, D. S., Aguilar, G., et al. 2017, arXiv:1707.09322

Ai, Y. L., Wu, X.-B., Yang, J., et al. 2016, AJ, 151, 24
Antonucci, R. 1993, ARA\&A, 31, 473

Antonucci, R. R. J., \& Cohen, R. D. 1983, ApJ, 271, 564

Antonucci, R. R. J., \& Miller, J. S. 1985, ApJ, 297, 621

Ballo, L., Giustini, M., Schartel, N., et al. 2008, A\&A, 483, 137

Bianchi, S., Guainazzi, M., Matt, G., et al. 2005, A\&A, 442, 185

Blanchard, P. K., Nicholl, M., Berger, E., et al. 2017, ApJ, 843, 106

Bolton, A. S., Schlegel, D. J., Aubourg, É., et al. 2012, AJ, 144, 144 Bruzual, G., \& Charlot, S. 2003, MNRAS, 344, 1000

Calderone, G., Nicastro, L., Ghisellini, G., et al. 2017, MNRAS, 472, 4051

Chambers, K. C., Magnier, E. A., Metcalfe, N., et al. 2016, arXiv: 1612.05560

Cohen, R. D., Puetter, R. C., Rudy, R. J., Ake, T. B., \& Foltz, C. B. 1986, ApJ, 311,135

Cui, X.-Q., Zhao, Y.-H., Chu, Y.-Q., et al. 2012, RAA, 12, 1197

Dawson, K. S., Schlegel, D. J., Ahn, C. P., et al. 2013, AJ, 145, 10

Denney, K. D., De Rosa, G., Croxall, K., et al. 2014, ApJ, 796, 134

Dey, A., Schlegel, D. J., Lang, D., et al. 2018, AJ, submitted (arXiv:1804.08657)

Drake, A. J., Djorgovski, S. G., Mahabal, A., et al. 2009, ApJ, 696, 870

Du, B., Luo, A.-L., Kong, X., et al. 2016, ApJS, 227, 27

Eisenstein, D. J., Weinberg, D. H., Agol, E., et al. 2011, AJ, 142, 72

Elitzur, M. 2012, ApJL, 747, L33

Elitzur, M., Ho, L. C., \& Trump, J. R. 2014, MNRAS, 438, 3340

Eracleous, M., Livio, M., Halpern, J. P., \& Storchi-Bergmann, T. 1995, ApJ, 438,610

Fan, Z., Wang, H., Jiang, X., et al. 2016, PASP, 128, 115005

Flaugher, B. 2005, IJMPA, 20, 3121

Fukugita, M., Ichikawa, T., Gunn, J. E., et al. 1996, AJ, 111, 1748

Gezari, S., Hung, T., Cenko, S. B., et al. 2017, ApJ, 835, 144

Guillochon, J., \& Ramirez-Ruiz, E. 2013, ApJ, 767, 25

Gunn, J. E., Siegmund, W. A., Mannery, E. J., et al. 2006, AJ, 131, 2332

He, B., Fan, D., Cui, C., et al. 2016, arXiv:1601.02334

Hönig, S. F., \& Kishimoto, M. 2017, ApJL, 838, L20

Hutchinson, T. A., Bolton, A. S., Dawson, K. S., et al. 2016, AJ, 152, 205

Hutsemékers, D., Agís González, B., Sluse, D., Ramos Almeida, C., \& Acosta Pulido, J.-A. 2017, A\&A, 604, L3

Ichikawa, K., Ricci, C., Ueda, Y., et al. 2017, ApJ, 835, 74

Ivezic, Z., Tyson, J. A., Abel, B., et al. 2008, arXiv:0805.2366

Khachikian, E. Y., \& Weedman, D. W. 1971, ApJL, 164, L109

Korista, K. T., \& Goad, M. R. 2004, ApJ, 606, 749

Kormendy, J., \& Ho, L. C. 2013, ARA\&A, 51, 511

LaMassa, S. M., Cales, S., Moran, E. C., et al. 2015, ApJ, 800, 144

Law, N. M., Kulkarni, S. R., Dekany, R. G., et al. 2009, PASP, 121, 1395

Lodato, G., King, A. R., \& Pringle, J. E. 2009, MNRAS, 392, 332

Luo, A.-L., Zhang, H.-T., Zhao, Y.-H., et al. 2012, RAA, 12, 1243

Luo, A.-L., Zhao, Y.-H., Zhao, G., et al. 2015, RAA, 15, 1095

Lyutyj, V. M., Oknyanskij, V. L., \& Chuvaev, K. K. 1984, SvAL, 10, 335

MacLeod, C. L., Ross, N. P., Lawrence, A., et al. 2016, MNRAS, 457, 389

MacLeod, C. L., Ivezić, Ž., Sesar, B., et al. 2012, ApJ, 753, 106

Mainzer, A., Bauer, J., Cutri, R. M., et al. 2014, ApJ, 792, 30

Mainzer, A., Grav, T., Bauer, J., et al. 2011, ApJ, 743, 156

Marchese, E., Braito, V., Della Ceca, R., Caccianiga, A., \& Severgnini, P. 2012, MNRAS, 421, 1803

Marin, F. 2017, A\&A, 607, A40

Martini, P., \& Schneider, D. P. 2003, ApJL, 597, L109

Matt, G., Guainazzi, M., \& Maiolino, R. 2003, MNRAS, 342, 422

McElroy, R. E., Husemann, B., Croom, S. M., et al. 2016, A\&A, 593, L8

Merloni, A., Dwelly, T., Salvato, M., et al. 2015, MNRAS, 452, 69

Moran, E. C., Halpern, J. P., \& Helfand, D. J. 1996, ApJS, 106, 341

Nenkova, M., Sirocky, M. M., Ivezić, Ž., \& Elitzur, M. 2008a, ApJ, 685, 147

Nenkova, M., Sirocky, M. M., Nikutta, R., Ivezić, Ž., \& Elitzur, M. 2008b, ApJ, 685, 160

Netzer, H. 2015, ARA\&A, 53, 365

Osterbrock, D. E. 1977, ApJ, 215, 733

Osterbrock, D. E. 1981, ApJ, 249, 462

Osterbrock, D. E., \& Koski, A. T. 1976, MNRAS, 176, 61P 
Pâris, I., Petitjean, P., Ross, N. P., et al. 2017, A\&A, 597, A79

Penston, M. V., \& Perez, E. 1984, MNRAS, 211, 33P

Piconcelli, E., Fiore, F., Nicastro, F., et al. 2007, A\&A, 473, 85 Rees, M. J. 1988, Natur, 333, 523

Ricci, C., Bauer, F. E., Arevalo, P., et al. 2016, ApJ, 820, 5

Risaliti, G., Miniutti, G., Elvis, M., et al. 2009, ApJ, 696, 160

Ruan, J. J., Anderson, S. F., Cales, S. L., et al. 2016, ApJ, 826, 188

Ruan, J. J., Anderson, S. F., Dexter, J., \& Agol, E. 2014, ApJ, 783, 105

Rumbaugh, N., Shen, Y., Morganson, E., et al. 2018, ApJ, 854, 160

Runnoe, J. C., Cales, S., Ruan, J. J., et al. 2016, MNRAS, 455, 1691

Schmidt, K. B., Rix, H.-W., Shields, J. C., et al. 2012, ApJ, 744, 147

Schneider, D. P., Richards, G. T., Hall, P. B., et al. 2010, AJ, 139, 2360

Seyfert, C. K. 1943, ApJ, 97, 28

Shangguan, J., Ho, L. C., \& Xie, Y. 2018, ApJ, 854, 158

Shapovalova, A. I., Popović, L. Č., Burenkov, A. N., et al. 2010, A\&A, 509, A106

Shappee, B. J., Prieto, J. L., Grupe, D., et al. 2014, ApJ, 788, 48

Sheng, Z., Wang, T., Jiang, N., et al. 2017, ApJL, 846, L7

Skrutskie, M. F., Cutri, R. M., Stiening, R., et al. 2006, AJ, 131, 1163

Smee, S. A., Gunn, J. E., Uomoto, A., et al. 2013, AJ, 146, 32

Stern, J., \& Laor, A. 2012, MNRAS, 426, 2703

Stoughton, C., Lupton, R. H., Bernardi, M., et al. 2002, AJ, 123, 485
Taylor, M. B. 2005, in ASP Conf. Ser. 347, Astronomical Data Analysis Software and Systems XIV, ed. P. Shopbell, M. Britton, \& R. Ebert (San Francisco, CA: ASP), 29

Tody, D. 1986, Proc. SPIE, 627, 733

Tody, D. 1993, in ASP Conf. Ser. 52, Astronomical Data Analysis Software and Systems II, ed. R. J. Hanisch, R. J. V. Brissenden, \& J. Barnes (San Francisco, CA: ASP), 173

Urry, C. M., \& Padovani, P. 1995, PASP, 107, 803

Vanden Berk, D. E., Richards, G. T., Bauer, A., et al. 2001, AJ, 122, 549

Vanden Berk, D. E., Wilhite, B. C., Kron, R. G., et al. 2004, ApJ, 601, 692

Wang, J.-M., \& Zhang, E.-P. 2007, ApJ, 660, 1072

Wang, S., Li, A., \& Jiang, B. W. 2015, MNRAS, 454, 569

Weingartner, J. C., \& Draine, B. T. 2001, ApJ, 548, 296

Wilhite, B. C., Vanden Berk, D. E., Kron, R. G., et al. 2005, ApJ, 633, 638

Wright, E. L., Eisenhardt, P. R. M., Mainzer, A. K., et al. 2010, AJ, 140, 1868

Wu, X.-B., Zhang, W., \& Zhou, X. 2004, ChJAA, 4, 17

Yang, Q., Wu, X.-B., Fan, X., et al. 2017, AJ, 154, 269

Zhao, G., Zhao, Y.-H., Chu, Y.-Q., Jing, Y.-P., \& Deng, L.-C. 2012, RAA, 12,723

Zou, H., Zhang, T., Zhou, Z., et al. 2017, AJ, 153, 276

Zuo, W., Wu, X.-B., Liu, Y.-Q., \& Jiao, C.-L. 2012, ApJ, 758, 104 